\title{
Review Article \\ Sterol Biosynthesis Pathway as Target for Anti-trypanosomatid Drugs
}

\author{
Wanderley de Souza ${ }^{1,2}$ and Juliany Cola Fernandes Rodrigues ${ }^{1}$ \\ ${ }^{1}$ Laboratório de Ultraestrutura Celular Hertha Meyer, Instituto de Biofísicia Carlos Chagas Filho, \\ Universidade Federal do Rio de Janeiro, CCS-Bloco G, 21941-902, Rio de Janeiro, RJ, Brazil \\ ${ }^{2}$ Diretoria de Programas, Instituto Nacional de Metrologia, Normalização e Qualidade Industrial, Rio de Janeiro, RJ, Brazil
}

Correspondence should be addressed to Wanderley de Souza, wsouza@biof.ufrj.br

Received 30 March 2009; Accepted 27 April 2009

Recommended by Herbert B. Tanowitz

\begin{abstract}
Sterols are constituents of the cellular membranes that are essential for their normal structure and function. In mammalian cells, cholesterol is the main sterol found in the various membranes. However, other sterols predominate in eukaryotic microorganisms such as fungi and protozoa. It is now well established that an important metabolic pathway in fungi and in members of the Trypanosomatidae family is one that produces a special class of sterols, including ergosterol, and other 24-methyl sterols, which are required for parasitic growth and viability, but are absent from mammalian host cells. Currently, there are several drugs that interfere with sterol biosynthesis (SB) that are in use to treat diseases such as high cholesterol in humans and fungal infections. In this review, we analyze the effects of drugs such as (a) statins, which act on the mevalonate pathway by inhibiting HMGCoA reductase, (b) bisphosphonates, which interfere with the isoprenoid pathway in the step catalyzed by farnesyl diphosphate synthase, (c) zaragozic acids and quinuclidines, inhibitors of squalene synthase (SQS), which catalyzes the first committed step in sterol biosynthesis, (d) allylamines, inhibitors of squalene epoxidase, (e) azoles, which inhibit C14 $\alpha$-demethylase, and (f) azasterols, which inhibit $\Delta^{24(25)}$-sterol methyltransferase (SMT). Inhibition of this last step appears to have high selectivity for fungi and trypanosomatids, since this enzyme is not found in mammalian cells. We review here the IC50 values of these various inhibitors, their effects on the growth of trypanosomatids (both in axenic cultures and in cell cultures), and their effects on protozoan structural organization (as evaluted by light and electron microscopy) and lipid composition. The results show that the mitochondrial membrane as well as the membrane lining the protozoan cell body and flagellum are the main targets. Probably as a consequence of these primary effects, other important changes take place in the organization of the kinetoplast DNA network and on the protozoan cell cycle. In addition, apoptosis-like and autophagic processes induced by several of the inhibitors tested led to parasite death.
\end{abstract}

Copyright (C) 2009 W. de Souza and J. C. F. Rodrigues. This is an open access article distributed under the Creative Commons Attribution License, which permits unrestricted use, distribution, and reproduction in any medium, provided the original work is properly cited.

\section{Introduction}

Sterols are constituents of the cellular membranes that are essential for their normal structure and function. In mammalian cells, cholesterol is the main sterol found in the various membranes. However, other sterols predominate in eukaryotic microorganisms such as fungi and protozoa. In the case of trypanosomatids, for many years cholesterol was considered to be the major sterol. This was due to the fact that all biochemical analyses were undertaken in protozoa grown in complex media containing either brain, heart, or liver extracts and bovine serum. When the first biochemical analysis of lower trypanosomatids grown in chemically defined medium was carried out, it became clear that they synthesize ergosterol and not cholesterol [1]. Subsequently, it was shown that the trypanosomatids incorporate cholesterol from the culture medium or from the blood of infected animals through a typical endocytic process involving, in the case of epimastigotes of Trypanosoma cruzi, the formation of endocytic vesicles in the flagellar pocket and the cytostome $[2,3]$.

It is now well established that an important metabolic pathway in fungi and in members of the Trypanosomatidae family is the sterol biosynthesis (SB) pathway. In these 


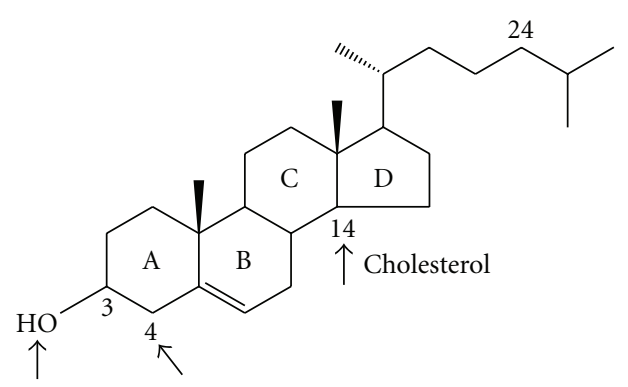

(a)

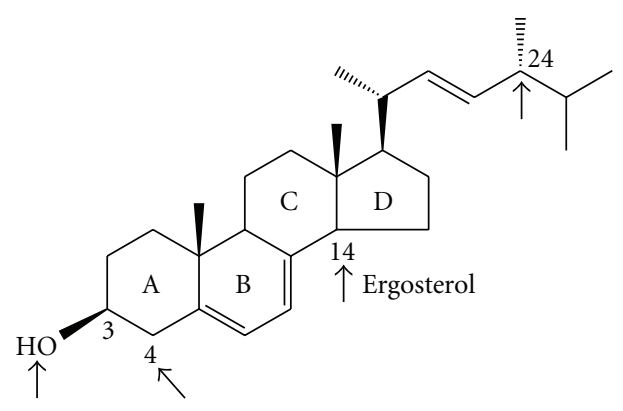

(b)

Figure 1: Molecular structures of cholesterol and ergosterol. The arrows indicate the parts of the molecules which have been shown to be essential for the growth of mammalian cells (cholesterol), fungi, and trypanosomatids (ergosterol and 24-methyl sterols).

organisms, this pathway produces a special class of sterols, including ergosterol and other 24-methyl sterols, that is, required for their growth and viability, but is absent from mammalian host cells $[4,5]$. Cholesterol and ergosterol differ in a few minor ways, as shown in Figure 1; cholesterol has only one double bond $(\Delta 5(6))$ in the $\mathrm{B}$ ring and has a fully saturated side chain without a methyl group at C24. It has been shown that some parts of the sterol molecule are important for its activity in cellular membranes. In the tetracyclic nucleus, the $3 \beta-\mathrm{OH}$ is obligatory for growth, whereas the presence of methyl groups at $\mathrm{C} 14$ or $\mathrm{C} 4$ do not allow growth. These two characteristics are essential for both cholesterol and ergosterol to support growth. However, certain characteristics of ergosterol that are absent in the cholesterol molecule, such as the presence of two double bonds in the $\mathrm{B}$ ring of the steroid nucleus, the presence of a $\beta$ methyl at position 24 , and the double bond at C22 in the side chain are essential for the growth of fungi and trypanosomatids.

The main sterols of the trypanosomatids comprise $\Delta^{5,7}$ compounds belonging to the $\mathrm{C}_{28}$-ergostane (24-methyl or methylene) or the $\mathrm{C}_{29}$-stigmastane (24-ethyl or ethylidine) groups [6-9]. In Leishmania amastigotes and promastigotes, the predominant sterol includes ergosta-5,7,24( $\left.24^{1}\right)$-trien$3 \beta$-ol (5-dehydroepisterol), although ergosta- $1187,24\left(24^{1}\right)$ dien-3 $\beta$-ol (episterol) and ergosta-5,7,22-trien-3 $\beta$-ol (ergosterol, the major fungal sterol) are present in smaller amounts. Stigmastane-related sterols comprise $5 \%$ of the total sterols in promastigotes; which can go as high as $20 \%$ in amastigotes of some species [9-12]. In contrast, T. cruzi epimastigotes

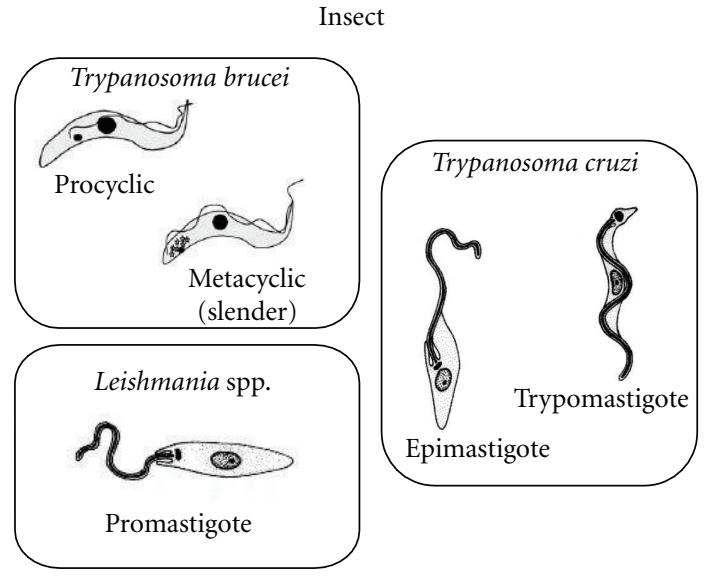

(a)

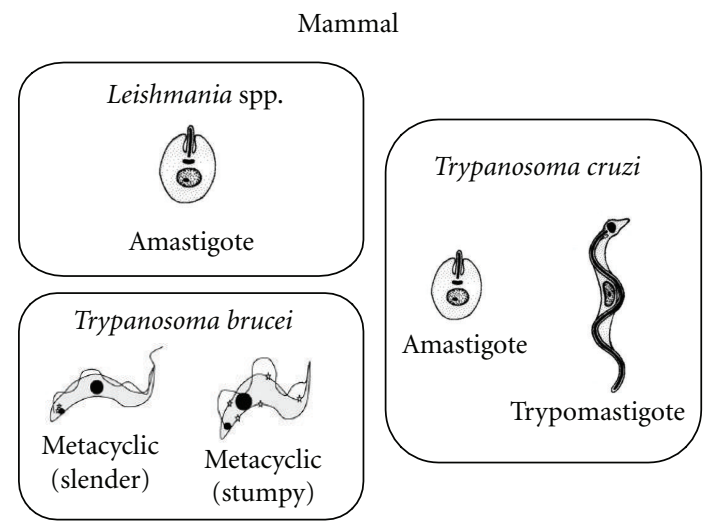

(b)

FIGURE 2: Schematic representation of main morphologies found during the life cycle of some members of the Trypanosomatidae family in the invertebrate host (insect) and vertebrate host (mammal).

contain around $40 \%$ ergosterol and ergosta-5,7-dien-3 $\beta$-ol, and an appreciable amount, around $30 \%$, of stigmasta-5,7dien-3 $\beta$-ol, and stigmasta-5,7,22-trien- $3 \beta$-ol $[8,13]$. In the case of amastigote forms, the sterol content is completely different since T. cruzi apparently does not produce $\Delta^{5,7}$. sterols, which are replaced by ergosta-7-en-3 3 -ol and 24 ethylidinecholest-7-en-3 $\beta$-ol, indicating the absence of $\Delta^{5}$ desaturase activity [14]. The content of sterols in Trypanosoma brucei differs from other trypanosomatids, where the bloodstream form contains predominantly cholesterol incorporated from the medium through a receptor-mediated endocytic process, which apparently suppresses de novo synthesis of $\mathrm{C}_{28}$-sterols [15], even though it has mRNA coding for enzymes involved in ergosterol biosynthesis [16]. In the procyclic form, the sterol content is different from the bloodstream form, with the major component in the total membranes being ergosterol, with some amount of cholesterol [16].

In the context of this review describing the effect of sterol biosynthesis inhibitors in members of the Trypasonomatidae family, it is important to show a schematic representation 


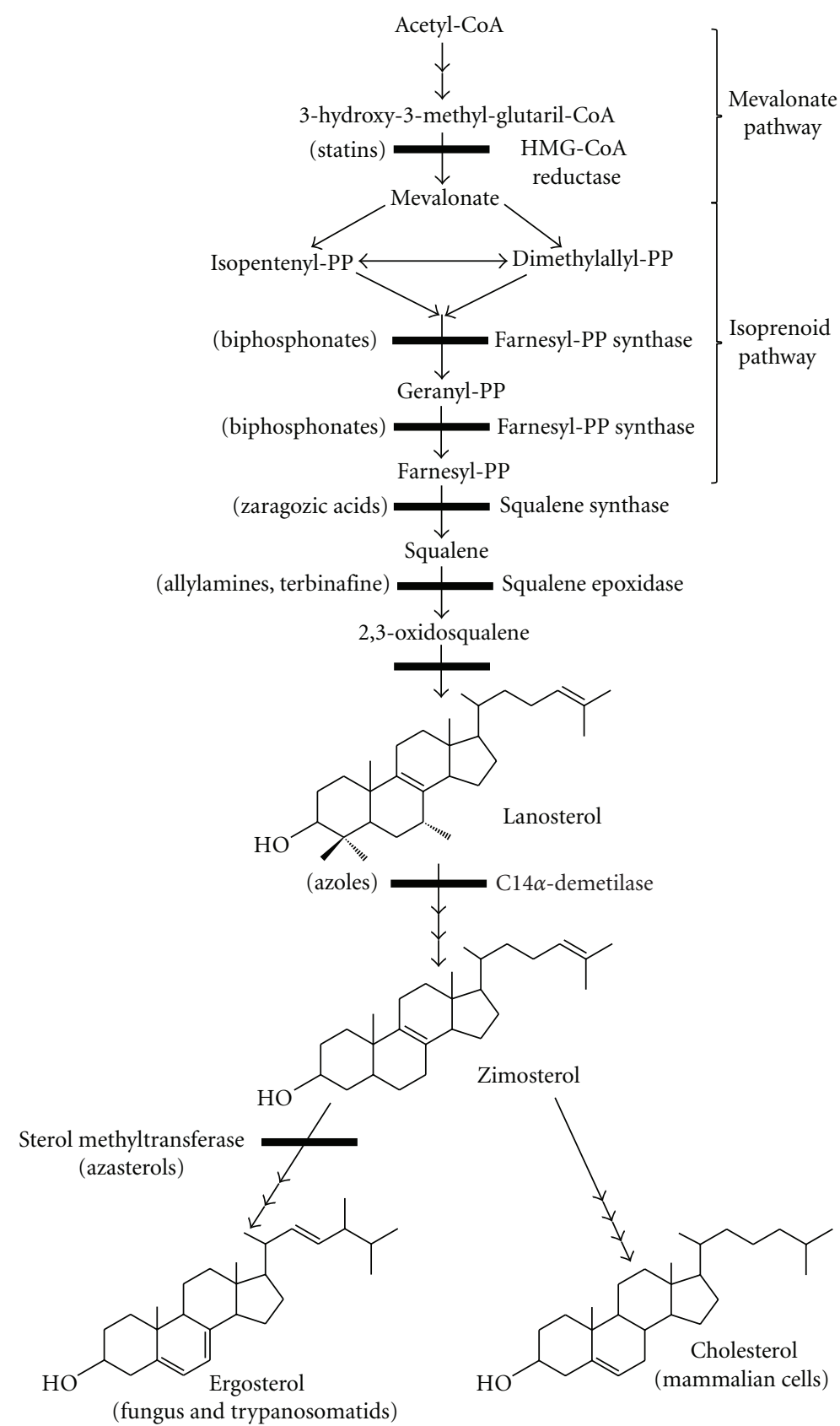

FIGURE 3: The biosynthesis of ergosterol and cholesterol showing the main steps, the enzymes involved, and the known inhibitors.

of the main developmental stages found in the invertebrate host (insect) and vertebrate host (mammal) for Trypanosoma brucei, Trypanosoma cruzi, and Leishmania spp (Figure 2) [17-20].

\section{Comparison of the Sterol Biosynthesis Pathways in Mammals, Fungi, and Trypanosomatids}

A comparative schematic diagram of the sterol biosynthesis pathway in eukaryotes is shown in Figure 3. At least 20 metabolic steps are necessary to synthesize such sterols as cholesterol and ergosterol, with some steps involving specific enzymes that differ between mammalian cells and microorganisms such as fungi and trypanosomatids. Some of these enzymes have been extensively studied, both as targets for the development of new drugs that interfere with parasite growth without severe effects on host cells and also as a means of reducing the high levels of endogenous cholesterol in mammalian cells.

The carbon skeleton of the sterol molecule is derived from acetyl-CoA, with the exception to the presence of the C24 methyl group in the ergosterol side chain. The first 
reactions in the biosynthetic pathway involve condensation of two acetyl-CoA units to form acetoacetyl-CoA, followed by the addition of a third unit to form 3-hydroxy-3methylglutaryl-CoA (HMG-CoA), which is then reduced by NADPH to give mevalonic acid. These three steps comprise the mevalonate pathway and are catalyzed by the cytosolic enzyme acetoacetyl-CoA thiolase, and two mitochondrial enzymes in yeasts and trypanosomatids, HMG-CoA synthase, and HMG-CoA reductase [21-23]. By contrast, the initial steps of cholesterol biosynthesis in mammalian cells occur mainly in the cytosol, with the later steps occurring in the endoplasmic reticulum (ER). On the other hand, several of the enzymes have been reported to exist in peroxisomes and in glycosomes [23-25].

After the mevalonate pathway, the next steps constitute the isoprenoid pathway. Isoprenoids are the most diverse and abundant compounds present in nature, and are essential components of all organisms due to a variety of roles in different biological processes. First, mevalonate is converted to isopentenyl diphosphate (IPP) by two phosphorylation reactions followed by one decarboxylation. Subsequently, isomerization of IPP by isopentenyl diphosphate isomerase produces dimethylallyl diphosphate (DMAPP). After that, longer isoprenoids are formed by a consecutive condensation of IPP with DMAPP and geranyl diphosphate (GPP) to produce the 15-carbon isoprenoid compound known as farnesyl diphosphate (FPP) in two reactions catalyzed by the enzyme farnesyl diphosphate synthase (FPPS). All these reactions together constitute the isoprenoid pathway, which is present in almost all organisms investigated so far [25]. FPP, which is the last product of isoprenoid biosynthesis, is the substrate for enzymes catalyzing the first committed step for the biosynthesis of sterols, ubiquinones, dolichols, heme A, and prenylated proteins. The enzymes involved in the isoprenoid pathway are located in different subcellular compartments such as the cytosol [26], mitochondria [27, 28], plastids in plant cells [26], and the peroxisome in animals [29]. For example, in Leishmania major, FPPS is located in the cytosol [30], while in Toxoplasma gondii it appears partially in the mitochondrion without any co-localization in rhoptries or apicoplast [31].

After the isoprenoid pathway, the next two reactions comprise the first committed step in sterol biosynthesis. These are catalyzed by the enzyme squalene synthase, which promotes a head-to-head condensation of two molecules of farnesyl diphosphate to produce squalene. In the first reaction, presqualene pyrophosphate (PPP) is produced by the loss of an inorganic pyrophosphate. This is converted to squalene in the second reaction in presence of NADPH, an essential cofactor required to drive this conversion [32]. In mammalian cells and in yeasts, squalene synthase is associated with the endoplasmic reticulum [33]. In L. major, its localization is still controversial. The presence of the signal sequences PTS1 and PTS2, typical of glycosomal proteins, indicates localization to the glycosome [34], while biochemical analysis of subcellular fractions of $T$. cruzi and Leishmania spp. showed that it could be found in the mitochondrion and microsomal fractions [35-37].
After production of squalene, sterol biosynthesis continues with the synthesis of 2,3-oxidosqualene (or squalene epoxide) in a reaction catalyzed by the enzyme squalene epoxidase. This is the first step in the conversion of the 30 -carbon chain squalene to the tetracyclic sterol skeleton. Squalene epoxidase is the first enzyme in the pathway that requires molecular oxygen, and this reaction is performed by a microsomal complex consisting of a flavoprotein with NADPH cytochrome $\mathrm{C}$ reductase activity, and a terminal oxidase that is not of the cytochrome P-450 family [38].

In a reaction that is considered to be one of the most complex in the sterol pathway, 2,2-oxidosqualene cyclase cyclizes the intermediate 2,3-oxidosqualene to lanosterol, the initial precursor of all steroid structures formed by mammals, fungi, and trypanosomatids.

After the cyclization of 2,3-oxidosqualene to form lanosterol, several sequential transformations occur to form cholesterol in mammals and ergosterol in trypanosomatids and fungi. Some of these reactions are common to all eukaryotes, whereas others are exclusive to each organism, with differences also between trypanosomatids and fungi.

One of the earliest steps in the lanosterol pathway is the demethylation of the ring system at the C14 position in a two-stage oxidative reaction catalyzed by a cytochrome P-450-containing monooxygenase enzyme known as lanosterol-14 $\alpha$-methyl demethylase (C14 $\alpha$-demethylase) [38].

Removal of the $14 \alpha$-methyl by C14 $\alpha$-demethylase generates a $\Delta^{8(14)}$ unsaturated sterol with a double bond at the C14 position. This unsaturation needs to be removed to produce $\Delta^{5}$ sterols in two consecutive reactions catalyzed by the enzyme $\Delta^{8(14)}$-reductase with NADPH as cofactor. In several fungi, this removal is essential, because the subsequent enzymes in the pathway do not metabolize this unnatural sterol. However in some fungal species such as Candida albicans [38, 40], and also L. amazonensis [11] and T. cruzi [42-44], it is metabolized by the C4-demethylase enzymes and $\Delta^{24}$-methenylase to form $\Delta^{14}$ fecosterol.

After removal of the C4 and C14 methyl groups and the methenylation of the side chain, the next reaction in the sequence is the isomerization of the double bonds in fecosterol in a reversible reaction catalyzed by the $\Delta^{7}-\Delta^{8}$ isomerase that does not require cofactors such as NADPH.

One of the most important stages of ergosterol biosynthesis that does not exist in the synthesis of cholesterol is the addition of a methyl group at the $\mathrm{C} 24$ position in the sterol side chain. Depending on the microorganism, this occurs early after the production of lanosterol, as observed in $L$. amazonensis [11], or at the level of zymosterol (which does not have the C4 and C14 methyl groups in the structure) as found in several fungi [45] and some Leishmania spp. [46]. The methyl group is transferred from S-adenosyl-Lmethionine (SAM) to C-24 of $\Delta^{24}$ sterols to produce $\Delta^{24(28)}$ sterols in some reactions catalyzed by the S-Adenosyl-Lmethionine: $\Delta^{24}$-sterol methyltransferase (EC 2.1.1.43; 24SMT). 24-SMT is a 150000 dalton membrane-bound protein that is present in plants, fungi, and trypanosomatids, but is absent in mammalian sterol biosynthetic systems; thus it may constitute an interesting target for the development 
of antifungal and antitrypanosomal agents. Cell fractionation studies have suggested that 24-SMT is located in the glycosomes and in the mitochondrion [35]. However, immunofluorescence and electron microscopic observations using antibodies generated against the recombinant protein showed that 24-SMT is located in the endoplasmic reticulum and in translucent vesicles that presumably belong to the endocytic pathway [47].

\section{Available Drugs that Interfere with the Sterol Biosynthetic Pathway}

Currently, there are several known drugs that interfere with sterol biosynthesis which are used to treat diseases such as high cholesterol in humans and fungal infections. Table 1 shows the representative compounds distributed in different classes, some of which are commercially available.

Statin is one of the main classes of the sterol biosynthesis inhibitors (SBIs), which act on the mevalonate pathway by the inhibition of HMG-CoA reductase. They have been widely used for cholesterol reduction in humans [38]. A drawback of the statins is their effect on the synthesis of isoprenoid compounds that are essential for several cellular events. Atorvastatin, widely used for treatment of hyperlipidemia, is one example of this class of drugs [48].

Bisphosphonate is another important class that interferes with the isoprenoid pathway inhibiting the step catalyzed by farnesyl diphosphate synthase (FPPS). They are used for the treatment of different bone resorption diseases, including osteoporosis, Paget's disease, hypercalcemia caused by malignancy, and tumor metastases in bone [49]. Alendronate and risedronate are two examples of bisphosphonates used for the treatment of osteoporosis and other bone resorption diseases [49].

Zaragozic acids and quinuclidines are known inhibitors of squalene synthase (SQS), which catalyzes the first committed step in sterol biosynthesis. This is a very attractive target because its inhibition does not interfere with isoprenoid production and intermediate metabolites that are formed can be readily metabolized and excreted [50]. SQS has been under intense scrutiny with the aim of developing new cholesterollowering agents for humans. Previous experimental studies with animals have demonstrated the effectiveness of quinuclidine-based SQS inhibitors such as 3-(biphenyl-4yl)-3-hydroxyquinuclidine [BPQ-OH] as cholesterol- and triglyceride-lowering agents [51-53].

Allylamines are known inhibitors of squalene epoxidase. One good example is terbinafine, which has been shown to be a potent compound against fungi, showing both oral and topical efficacy. Terbinafine inhibits squalene epoxidase leading to a depletion of ergosterol. Importantly, it does not inhibit the mammalian enzyme [38].

Azoles are important inhibitors of C $14 \alpha$-demethylase, and since they are effective against most fungal diseases, they are presently considered to be the most important antifungal compounds in use. Ketoconazole, one of the first azoles developed, was intensely used for several years. More recently, however, four new commercially available triazoles have been shown to be much more effective: fluconazole, itraconazole, voriconazol, and posaconazole.

The last class of ergosterol biosynthesis inhibitors comprises the azasterols, which inhibit $\Delta^{24(25)}$-sterol methyltransferase (SMT). Inhibition of this step appears to have high selectivity for fungi and trypanosomatids since this enzyme is not found in mammalian cells. Antifungal activities of azasterols have been described for Candida spp. [38, 39], Pneumocystis carinii [54], and Paracoccidioides brasiliensis [55].

In conclusion, based on the comments made above it is clear that further exploration of the sterol biosynthesis pathway is highly relevant to the treatment of both chronic diseases, such as hypercholesterolemia, and infectious diseases caused by fungi and trypanosomatids. Therefore, drugs known to inhibit enzymes of the sterol biosynthesis pathway should be tested against all these diseases. Indeed, several drugs developed either to reduce cholesterol levels in humans or to treat fungal diseases have been tested with some success against trypanosomatids, as will be discussed below. We also believe that new drugs shown to be active against trypanosomatids should be tested on fungi as well as in mammals.

\section{Effects of SB Inhibitors on Trypanosomatid Growth}

In T. cruzi, inhibitors of HMG-CoA reductase such as mevinolin (lovastatin) have been tested in vitro and in vivo. Against the extracellular proliferative epimastigote forms, mevinolin produced a dose-dependent reduction of the growth rate up to $25 \mu \mathrm{M}$. In the intracellular proliferative amastigote forms only very modest effects were observed up to $0.75 \mu \mathrm{M}$, above which a significant effect was also observed in the mammalian host cells. In intracellular amastigotes, it has been shown that supplementation of mevinolin with ketoconazole gives a synergistic effect, so that lower concentrations of azoles become more effective. On the other hand, the combination of mevinolin with terbinafine produced an additive effect, whereas the combination of mevinolin, terbinafine, and ketoconazole showed again a synergistic effect on amastigotes [56]. These results together indicate that a combination of drugs acting in consecutive steps of the sterol biosynthesis pathway may be a promising approach for the treatment of diseases caused by some pathogenic protozoa.

Bisphosphonates have been tested in vivo and in vitro against different protozoan parasites, including Leishmania spp., T. cruzi, T. brucei, Plasmodium falciparum, and T. gondii [57-59]. More than 50 new compounds were tested in different protozoan parasites, some of them presenting $\mathrm{IC}_{50}$ values lower than $1 \mu \mathrm{M}[60,61]$. In addition, to confirm that the isoprenoid enzymes are involved in the inhibition, they were also tested on the isolated L. major, T. cruzi, and T. gondii farnesyl diphosphate synthase enzymes (LmFPPS, TcFPPS, and TgFPPS). A potent inhibition of the enzyme's activities indicated that some of them are specific for inhibition of the isoprenoid pathway, thus validating this pathway as an 
TABLE 1: Representative compounds that interfere with sterol synthesis in eukaryotes, fungi and trypanosomatids.

Class
Mechanism of action

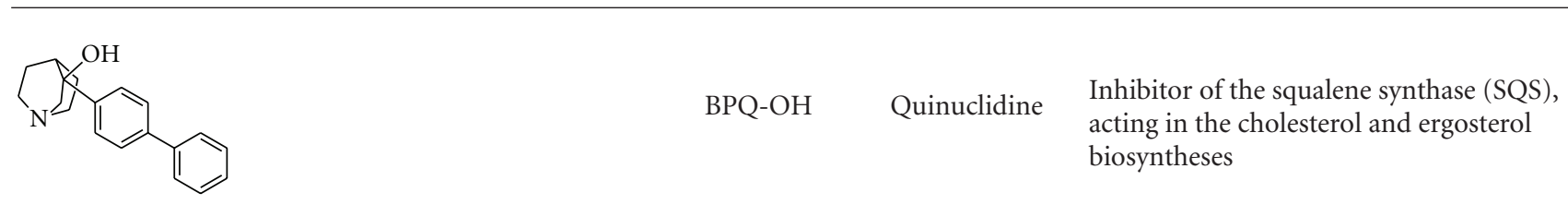<smiles>CN(C/C=C/C#CC(C)(C)C)Cc1cccc2ccccc12</smiles>

Terbinafine Allylamine Inhibitor of the squalene epoxidase. Used for a long time to treat fungal infections

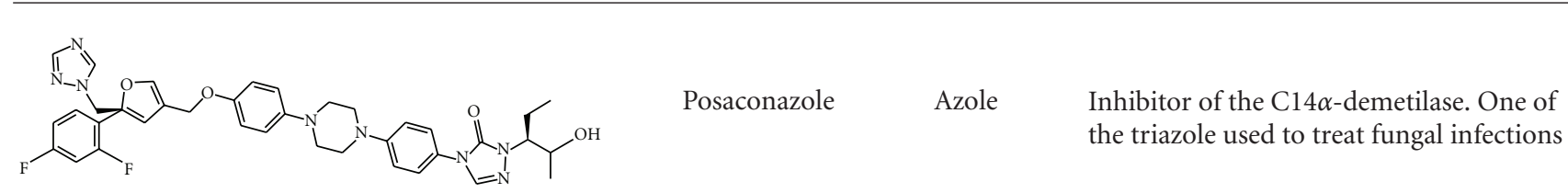<smiles>CC12CCC(O)CC1CCC1C2CCC2(C)C1CCC2[C@](C)(O)C1CCCN1</smiles>
22,26-azasterol Azasterol $\quad \begin{aligned} & \text { Inhibitor of the } \Delta^{24(25)} \text {-sterol } \\ & \text { methyltransferase, one enzyme presents } \\ & \text { exclusively in the ergosterol biosynthesis }\end{aligned}$

interesting and selective drug target for these parasites. Garzoni et al. [62] reported that risedronate showed a selective effect against Trypanosoma cruzi, leading to complete growth arrest and lysis at $150 \mu \mathrm{M}$ for epimastigotes. Complete destruction of intracellular amastigotes was observed at $100 \mu \mathrm{M}$ risedronate, thus preventing the development of $T$. cruzi infections in murine muscle heart or in Vero cells [62].
In trypanosomatids and fungi, there are several works describing the potent and selective activity of zaragozic acids and quinuclidines [35-37, 63-68]. For example, ER-119884 and E5700, two novel quinuclidine derivatives produced by Eisai Co. (Tokyo, Japan), have been shown to be potent antiTrypanosoma and Leishmania agents in vitro, leading to a dramatic depletion of the parasite's endogenous sterols, that 
is, associated with an intense antiproliferative activity [36, 37]. Figures 4(a)-4(d) shows the antiproliferative effect of E5700 (Figures 4(a) and 4(c)) and ER-119884 (Figures 4(b) and $4(\mathrm{~d}))$ in promastigotes and intracellular amastigotes of Leishmania amazonensis. These compounds are very potent against both forms of the life cycle, presenting MIC values of 30 and $10 \mathrm{nM}$ for promastigote, and 2.0 and $0.5 \mathrm{nM}$ for intracellular amastigotes [37]. When compared with the minimal concentration that affects macrophages, these values are, respectively, 100000 and 25000 fold greater than the corresponding $\mathrm{IC}_{50}$, indicating that they are selective against the parasite without any effect in the host cells.

In Leishmania, Vannier-Santos et al. [70] showed that terbinafine is able to interfere with the growth of promastigotes and intracellular amastigotes, inducing dramatic changes in their structural organization, especially in the mitochondrion. For terbinafine, the $\mathrm{IC}_{50}$ values were around $1 \mu \mathrm{M}$ for promastigotes and $100 \mathrm{nM}$ for intracellular amastigotes. However when in combination with ketoconazole, which also interferes with ergosterol biosynthesis, the values decreased to around $1 \mathrm{nM}$ for intracellular amastigotes, indicating once more that the approach of inhibiting multiple steps of this pathway is a promising alternative for chemotherapy [70].

In trypanosomatids, azoles have been used in vitro against T. cruzi and Leishmania. Drugs such as D0870 and posaconazol were tested in vitro and in vivo with positive and interesting results, and also have a potent effect against acute and chronic experimental Chagas' disease [69, 7175]. Ketoconazole was also tested alone or in combination with other sterol biosynthesis inhibitors [42, 44, 71, 76]. The $\mathrm{IC}_{50}$ of Posaconazole for $T$. cruzi epimastigotes and amastigotes was 3 and $0.25 \mathrm{nM}$, respectively. However when in combination with amiodarone, $\mathrm{a} \mathrm{K}^{+}$and $\mathrm{Ca}^{2+}$ channel antagonist, they presented synergistic effects with a fractional inhibitory concentration (FIC) of $0.42 \mu \mathrm{M}$ [73]. Amiodarone was also tested in Leishmania mexicana, having an $\mathrm{IC}_{50}$ similar to that found for T. cruzi, due to alterations in the physiology of the mitochondrion and acidocalcisomes [77].

The effects of azasterols on T. cruzi [4, 42-44, 78], Leishmania [5, 11, 47, 78, 79], and T. brucei [16] have been extensively studied. The antifungal activities of azasterols against Candida spp., $P$. carinii [54], and $P$. brasiliensis [55] have also been described. We have shown that several azasterols are active against $L$. amazonesis with $\mathrm{IC}_{50} \mathrm{~s}$ in the submicromolar to nanomolar range, indicating that this step has potential as a chemotherapeutic target $[11,78,80]$. Furthermore, when in combination with azoles they are even more effective, and sometimes acting synergistically [43].

\section{Effects of SB Inhibitors on the Ultrastructure of Trypanosomatids}

It has been shown that ergosterol biosynthesis inhibitors induce dramatic alterations in the ultrastructure of several organelles $[11,37,64,66,70,74-76,78-81]$. These alterations occur mainly in the mitochondrion-kinetoplast complex, the endoplasmic reticulum, the Golgi complex, nucleus, multivesicular structures, lipidic inclusions, the contractile vacuole, and also in the plasma membrane covering the cell body, and the flagellum.

The mitochondrion-kinetoplast complex has been shown to constitute an important organelle target of drugs that inhibit sterol biosynthesis. It is important to point out that T. cruzi and Leishmania have only one highly ramified mitochondrion distributed throughout the protozoan body (Figure 5(a)) (reviewed in [82]). Treatment of L. amazonensis with different azasterols induced mitochondrion alterations such as a disorganization of the mitochondrial membranes (Figures 5(b)-5(c)) followed by an intense swelling and loss of the matrix contents (Figures 5(b)-5(d)) [11, 76]. These alterations in the mitochondrion were also observed after treatment of T. cruzi and L. amazonensis with terbinafine, ketoconazole, and ICI195,739 [70, 74-76]. The swelling is dramatic and the mitochondrion appears to occupy the whole cytosol in epimastigotes treated with ketoconazole (Figures 6(a)-6(b)). The mitochondrial alterations were confirmed by measuring the mitochondrial membrane potential in digitonin-permeabilized parasites [79]. On the other hand, the inhibitors did not affect the macrophage's mitochondria, which can be visualized using JC-1, a cationic mitochondrial vital dye [37]. Most probably all these morphological changes are due to modifications in the composition of the mitochondrial membranes due to interference with the synthesis of ergosterol and accumulation of intermediate metabolites [83]. Lipid analysis performed in epimastigotes of $T$. cruzi showed that the mitochondrion has a different lipid composition when compared with mammalian cells, including the presence of ergosterol, thus explaining the potent effect of SB inhibitors on its ultrastructure and physiology [84].

One characteristic feature of the unique mitochondrion of trypanosomatids is the presence of a complex network of DNA localized in a portion of the mitochondrion. This network is located just below the basal bodies from which the flagellum is formed, and is known as the kinetoplast (reviewed in [85]). The kinetoplast DNA is organized as thousands of concatenated minicircles and a few maxicircles. It was shown that after treatment with E5700, an SQS inhibitor, alterations were observed in the kinetoplast structure of L. amazonensis. In treated cells, the kinetoplast appeared completely disorganized relative to its normal structure (Figure 6(c)). These alterations probably result from changes that take place in the organization of the inner mitochondrial membrane, that is, connected to the kinetoplast DNA network.

Alterations in the nuclear membrane, Golgi complex and endoplasmic reticulum were also observed after treatment with different SB inhibitors (Figures 7(a)-7(c)). The presence of a multivesicular body associated with the trans-Golgi network can be seen in Figure 7(b) (star), thus suggesting alterations in the secretory pathway. Furthermore, Figure 7(c) (stars) also shows the presence of some vacuoles resembling autophagosomes, thus suggesting the occurrence of cell death by autophagy (reviewed in [86-88]). The presence of large vacuoles containing membrane profiles, endoplasmic reticulum forming myelin-like figures or engulfing a part of 


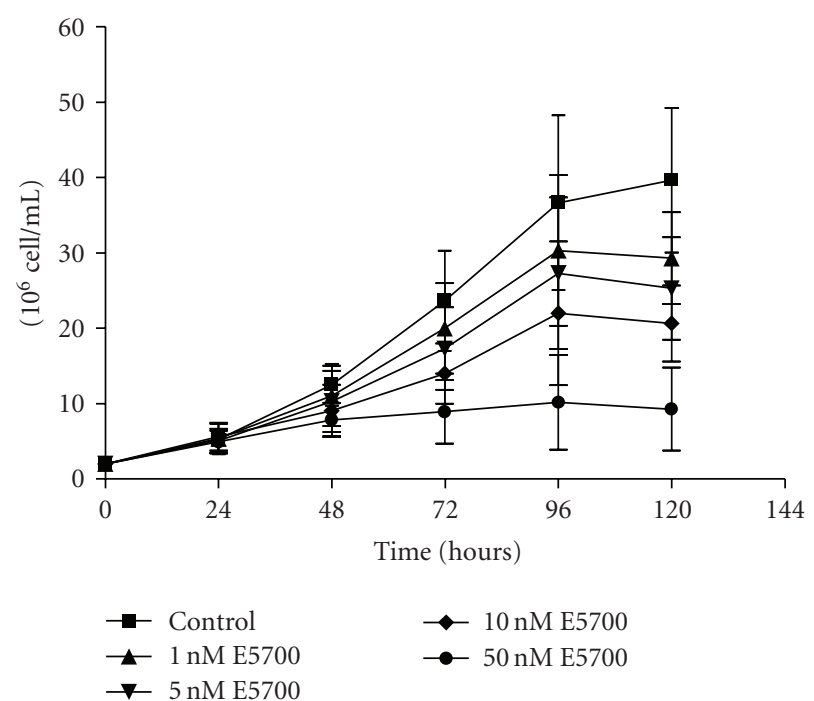

(a)
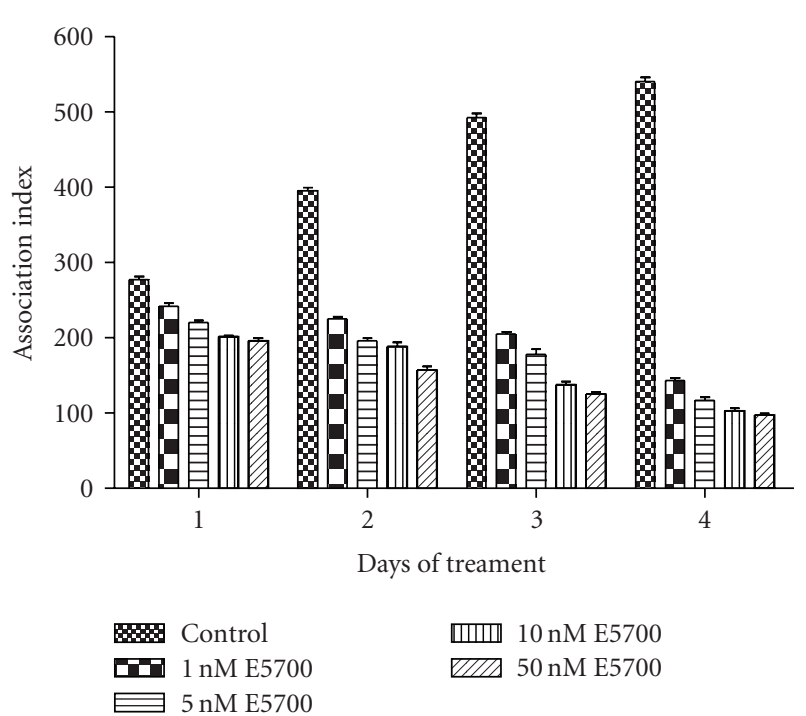

(c)

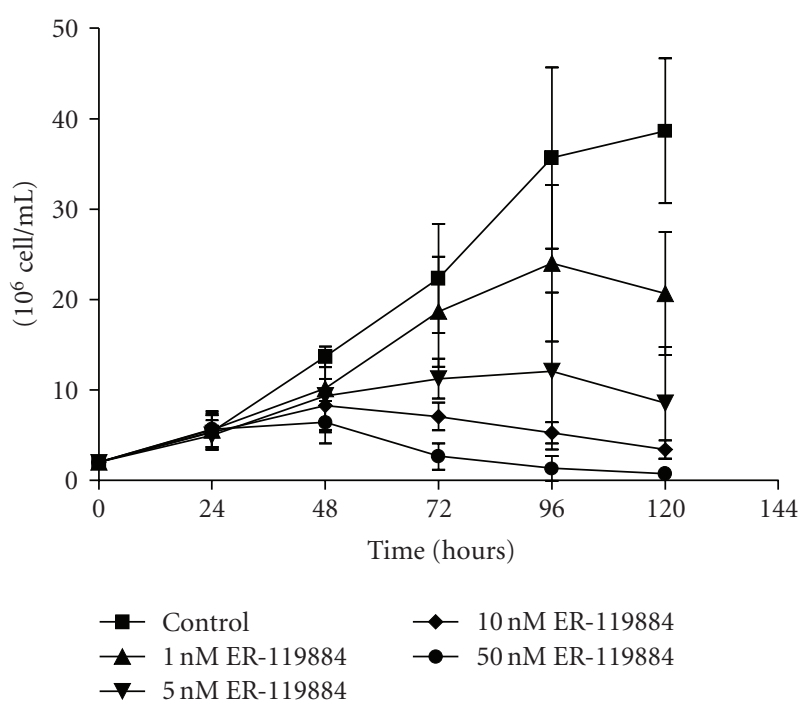

(b)
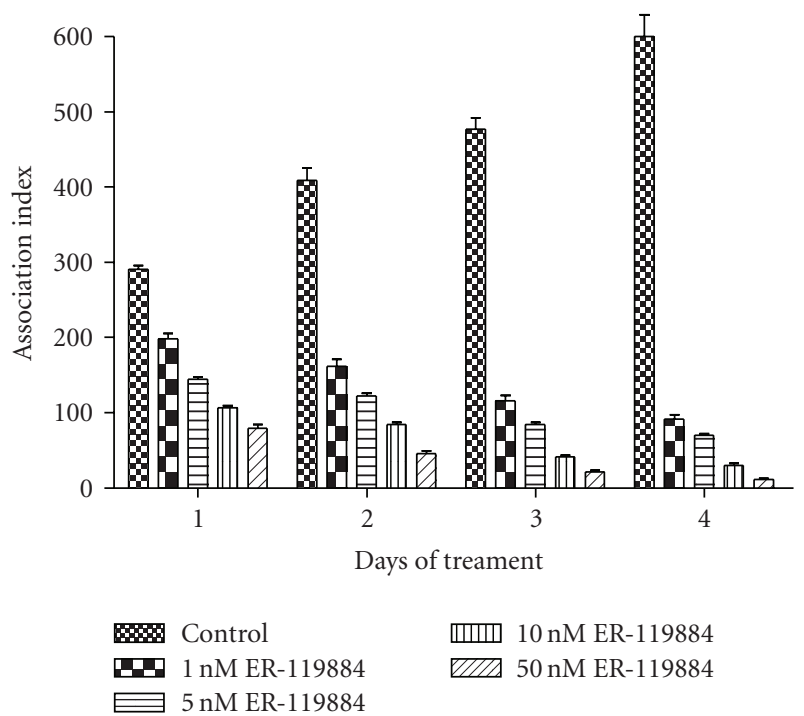

(d)

FIGURE 4: (a)-(b) Growth curves of promastigotes and (c)-(d) intracellular amastigotes of Leishmania amazonensis treated with two potent squalene synthase inhibitors, E5700 and ER-119884. The graphics are reproduced with permission from [37] American Society for Microbiology.

the cytoplasm, and damaged organelles supports the idea that an autophagy-like process takes place in these cells (Figures 8(a)-8(b)).

Recent studies using various microscopy techniques have shown that trypanosomatids possess a structure located close to the flagellar pocket identified as a contractile vacuole (reviewed in [89]). This structure became much more evident in L. amazonensis (Figures 9(a)-9(b)) and T. cruzi epimastigotes [64] treated with SB inhibitors. As observed by differential interference contrast microscopy, the treated cells appeared rounded and swollen (Figure 9(a)), suggesting osmotic changes, thus explaining the presence of a prominent contractile vacuole. These changes may be due to alterations in the plasma membrane's permeability to ions induced by the complete depletion of sterols and sterol-like molecules, which is likely to lead to significant changes of the physicochemical properties of the lipid bilayer [90].

Another important alteration observed after treatment with SB inhibitors is the presence of several lipid droplets displaying variable morphology, as shown in Figures 10(a)10(b). The formation of these structures is probably due to accumulation of lipid precursors. The images showed a large variation in the electron density of the structures, suggesting that different types of lipids are accumulated. Some appeared very dense after postfixation with osmium tetroxide (Figure 10(a)). Others, however, appeared as electronlucent structures (Figure 10(b), large arrows and stars). They are surrounded by a typical monolayer of phospholipids 


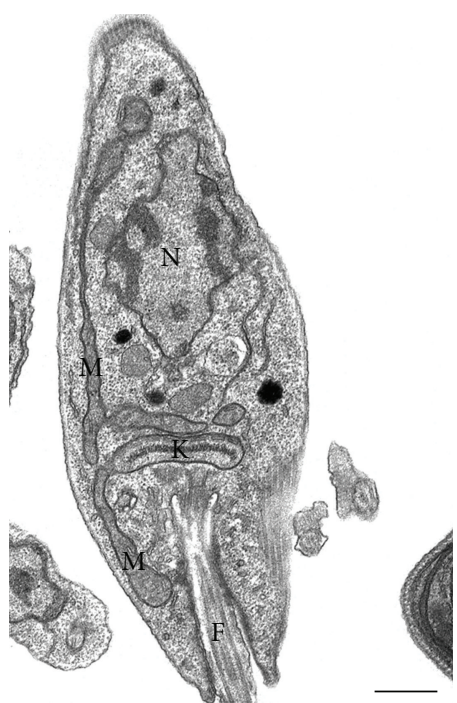

(a)

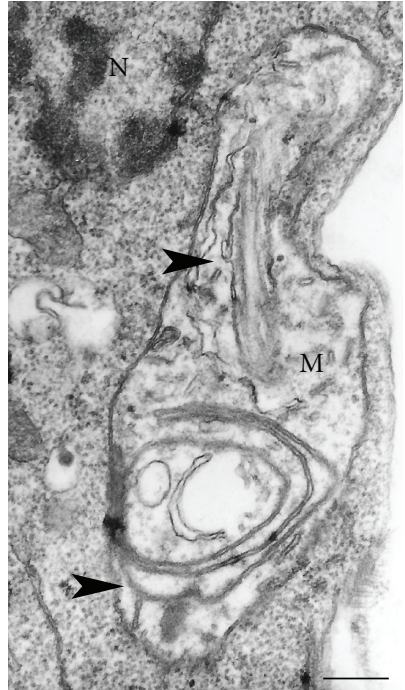

(b)

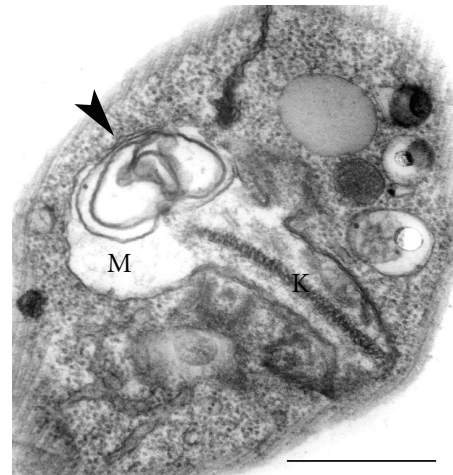

(c)

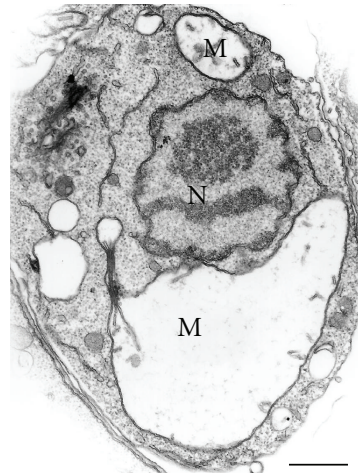

(d)

FIGURE 5: Ultrathin sections of L. amazonensis promastigotes control (a) and treated with azasterols, known inhibitors of the $\Delta^{24(25)}$-sterol methyltransferase (b)-(d). (a) General overview of an untreated-parasite showing a normal ultrastructure of the mitochondrion (M), kinetoplast (k), flagellum (F) and nucleus $(\mathrm{N})$. (b)-(d) Treated-parasites presenting severe alterations in the mitochondrion structure such as a disorganization of the internal membranes ((b) and (c), arrowheads) and an intense and evident mitochondrial swelling with loss of the matrix content (b) $-(\mathrm{d})$. Bars $0.5 \mu \mathrm{m}$.

(high magnification in Figure 10(b)). These lipid droplets could be also identified by fluorescence microscopy using the neutral lipid marker Nile Red [37]. Lipid droplets were also observed in L. major null mutants for important enzymes of the sphingolipid biosynthesis pathway [91, 92], and in T. cruzi after perturbation of the sphingolipid content [93]. On the other hand, they also accumulated when epimastigotes were treated with different classes of inhibitors, including cytoskeletal inhibitors [94], indicating that lipid body formation can occur as a consequence of perturbations in parasitic functions not related to lipid biosynthesis.

Alterations in the plasma membrane lining the cell body, flagellar pocket and flagellum were also observed, but the morphological changes varied according to the inhibitor (Figures 11(a)-11(b)). When the parasites where incubated with azasterols, alterations in the flagellar pocket were predominant (Figure 11(a)), while SQS inhibitors induced alterations mainly in the membrane lining the cell body (Figure 11(c)-11(d)) and sometimes in the flagellar membrane (Figure 11(b)) [64]. These different phenotypes suggest that the three domains of the plasma membrane have distinct lipid compositions. On the other hand, the presence of membrane blebs could be related to apoptosis-like death [86].

It is well known that in trypanosomatids there is a close connection between the plasma membrane lining the cell body and the subpellicular microtubules, and that the spatial distribution of these microtubules is responsible for the maintenance of the protozoan's shape (reviewed in [95]). Immunofluorescence microscopy of tubulin-stained trypanosomes treated with an SBI revealed changes in the shape of the cell and in the distribution of the subpellicular microtubules, probably due to alterations in the sterol composition of the plasma membrane [37]. Figures 12(a)12(d) show the effect of ER-119884, an SQS inhibitor, on the cytoskeleton of $L$. amazonensis promastigotes. The changes were also seen by transmission electron microscopy (Figure 11(d)).

Recent observations show that SBIs also interfere with the protozoan cell cycle. Using fluorescence microscopy of cells stained with DAPI to label the nucleus and kinetoplast, as well as transmission electron microscopy, it was shown that ER-119884 and BPQ-OH interfere dramatically with the cell cycle, inducing several abnormal phenotypes, including cells with multiple nuclei, kinetoplasts, and flagella (Figures 13(a)-13(d)). The effect on the cell cycle was already evident after 24 hours of incubation in the presence of the inhibitors, and the number of cells containing abnormal numbers of flagella, kinetoplasts, and nuclei increased with time [37]. There are at least two possible explanations for these effects: (i) the cells do not complete cell division due to the depletion of essential endogenous sterols, which control the dynamics of the membrane but are also key regulators of the cell cycle $[96,97]$, or (ii) the organization of the cytoskeleton necessary for the completion of cytokinesis, which requires interactions with the nuclear membrane, is in some way affected by SQS inhibitors. In addition, SBI also induced significant alterations in the trypanosomatids' nuclei.

A significant number of treated cells showed abnormal chromatin condensation (Figure 14), indicating a process of cell death by apoptosis (reviewed in [86-88]).

All these alterations discussed here indicate that sterols play an essential role in a significant number of cellular processes, including the cell cycle, cell death, and the 


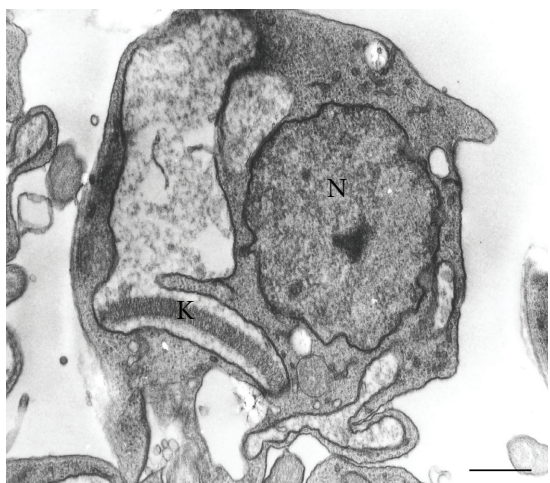

(a)

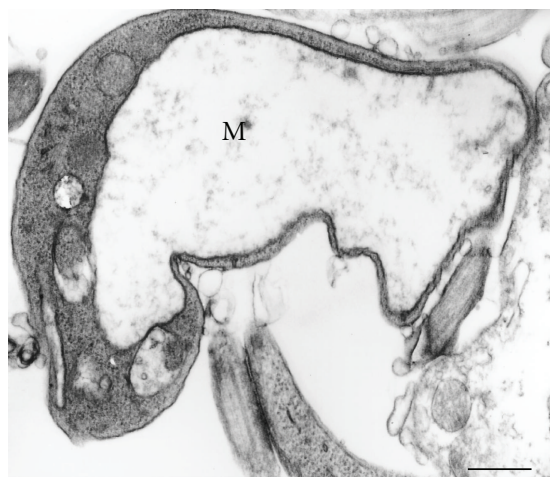

(b)

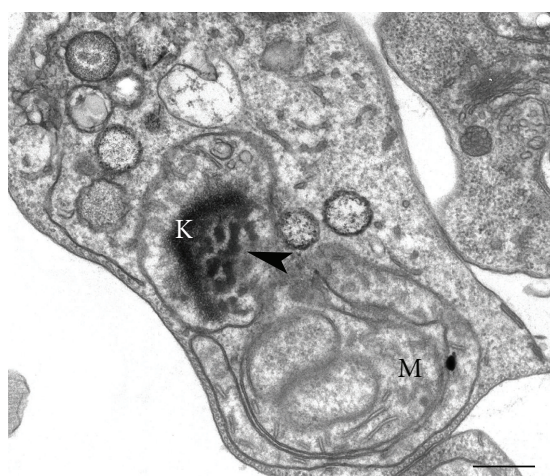

(c)

FIgURE 6: Trypanosoma cruzi epimastigotes treated with ketoconazole, an inhibitor of the C14 $\alpha$-demetilase (a)-(b), and $L$. amazonensis promastigotes treated with E5700, an inhibitor of the squalene synthase (c) showing an intense mitochondrial swelling (a)-(b) and alterations in the kinetoplast structure ((c), arrowhead). $\mathrm{K}$, kinetoplast; $\mathrm{M}$, mitochondrion. (a)-(b) Images are reproduced with permission from [74] American Society for Microbiology. Bars, $0.5 \mu \mathrm{m}$.

maintenance of the membrane's structure, stability, and function.

\section{Effects of SB Inhibitors on Lipid Composition}

It is now well established that SB inhibitors lead to the accumulation of intermediates of the ergosterol biosynthesis

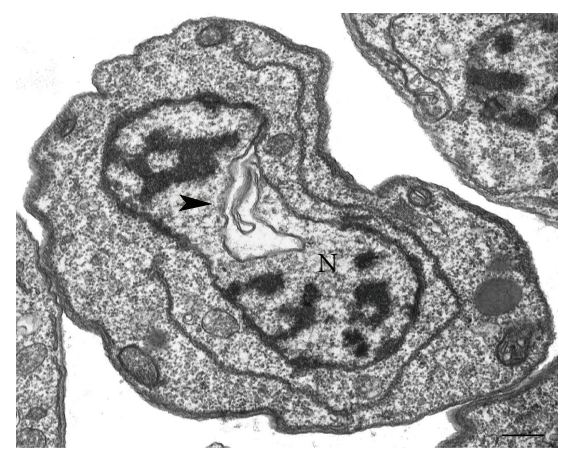

(a)

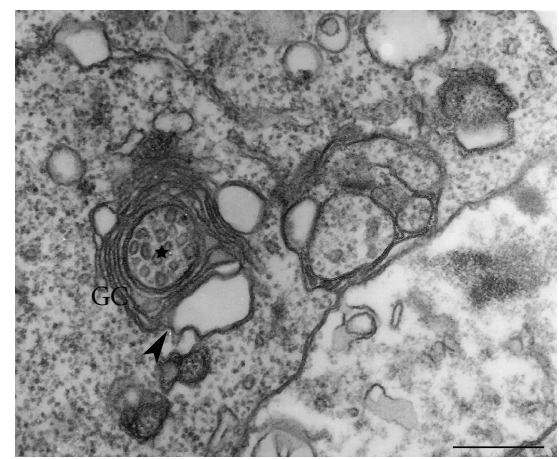

(b)

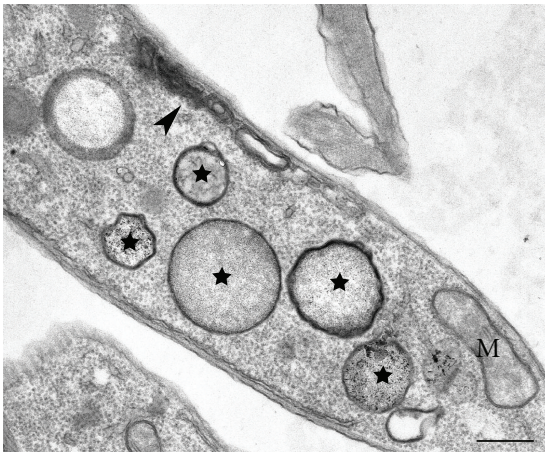

(c)

FIGURE 7: L. amazonensis promastigotes treated with azasterols (a)-(b), and ER-119884 (c) showing alterations in the nuclear membrane ((a), arrowhead), in the Golgi complex ((b), arrowhead), and in the endoplasmic reticulum ((c), arrowhead). In the Figures 6(b) and 6(c), the presence of a multivesicular bodies and autophagosome-like structures (stars) could be related with a remodeling process of damaged organelle by autophagy. GC, Golgi complex; M, mitochondrion; N, nucleus. Bars, $0.5 \mu \mathrm{m}$.

pathway (reviewed in [4]). Treatment of T. cruzi epimastigotes with mevinolin, an inhibitor of HMG-CoA reductase, led to a significant reduction in the presence of ergosterollike molecules, with a concomitant increase in exogenous cholesterol [56].

Incubation of the parasites with bisphosphonates, inhibitors of the farnesyl diphosphate synthase (FPPS), 


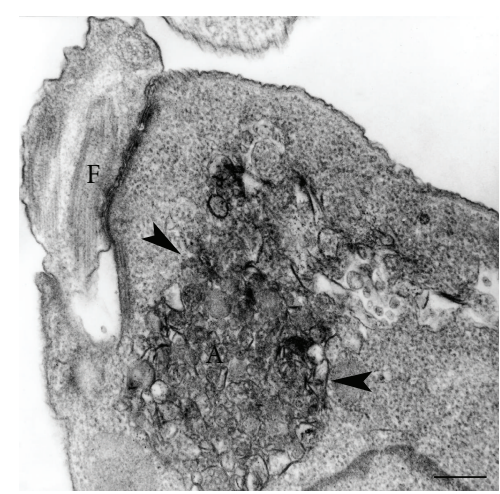

(a)

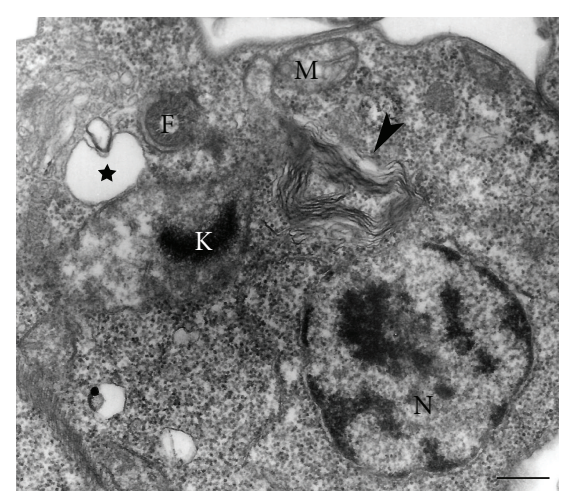

(b)

FIGURE 8: Promastigotes treated with quinuclidine and azasterol, respectively, showing the presence of structures related with autophagy such as a large vacuole containing many membrane profiles ((a), arrowheads), and a myelin-like figures involving part of the cytosol ((b), arrowhead). Star indicates the presence of a possible contractile vacuole near the flagellar pocket. A, autophagosome; F, flagellum; $\mathrm{K}$, kinetoplast; M, mitochondrion; N, nucleus. (a) This image is reproduced with permission from [66] Elsevier. Bars, $0.25 \mu \mathrm{m}$.

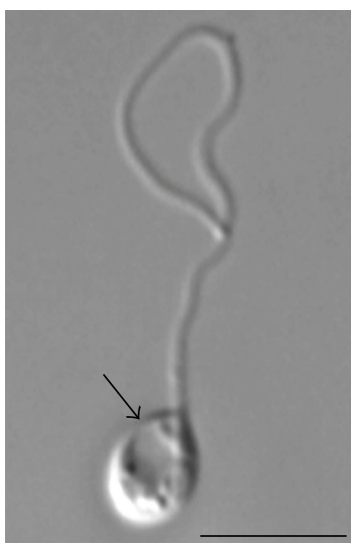

(a)

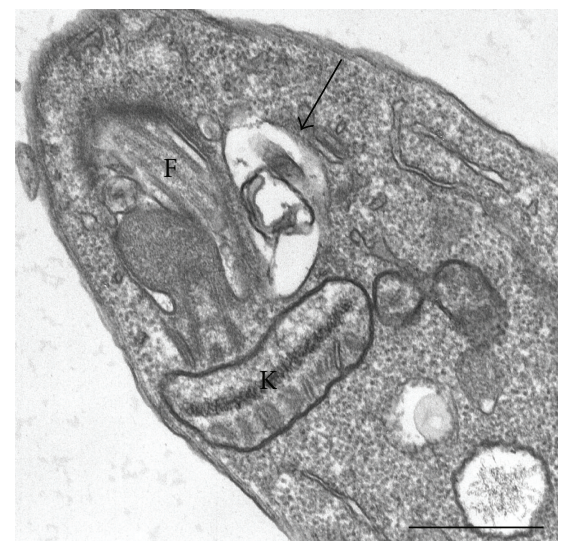

(b)

Figure 9: (a) Diferential interference contrast (DIC) microscopy and (b) transmission electron microscopy showing the presence of a prominent contractile vacuole (arrows) near the flagellar pocket after treatment of L. amazonensis promastigotes with quinuclidine inhibitors. In the left panel it is possible to observe a rounded and swollen parasite that probably indicates osmotic changes due alterations in the plasma membrane's permeability. F, flagellum; $\mathrm{K}$, kinetoplast. Bars, $5 \mu \mathrm{m}$ and $0.5 \mu \mathrm{m}$, respectively.

induces an accumulation of isopentenyl diphosphate (IPP) and inhibits the formation of farnesyl diphosphate (FPP). Consequences of this inhibition include a decrease in the level of protein prenylation, and reduced production of molecules such as dolichols, ubiquinones, heme a, and sterols (reviewed in [58]).

The inhibition of squalene synthase (SQS) induces a total depletion of squalene, endogenous ergosterol, and other 24-methyl sterols, and these are completely replaced by exogenous cholesterol [35-37]. This potent effect in the sterol composition is consistent with the inhibition of SQS enzyme in concentrations near the nanomolar to subnanomolar range, in a reaction that is noncompetitive with the substrate [37].

Treatment of L. mexicana promastigotes with terbinafine, a potent inhibitor of squalene-2,3-epoxidase, results in the accumulation of squalene, and a reduction in the amount of endogenous $\mathrm{C}_{28^{-}}$and $\mathrm{C}_{29}$-sterols [98]. In T. cruzi epimastigotes the same treatment led to the depletion of sterols and accumulation of phosphorylated hydrocarbons, with a lower amount of squalene when compared with Leishmania [99]. The $\mathrm{K}^{+}$and $\mathrm{Ca}^{+2}$ channel antagonist amiodarone also induces the inhibition of squalene epoxidase in T. cruzi and L. mexicana, leading to an accumulation of squalene and complete depletion of 24-methyl sterols such as ergosterol and 5-dehydroepisterol [73, 77].

Incubation of the parasites in the presence of azoles, which inhibit C14 $\alpha$-demethylase, led to the replacement of normal endogenous sterols by various $14 \alpha$-methyl sterols. In this situation, the first sterol that accumulates in promastigotes of Leishmania is $4 \alpha, 14 \alpha$-dimethyl-dimethylzymosterol; however, after a long exposure this sterol molecule could be metabolized by 24-SMT, producing sterols alkylated at C24 , or demethylated at C-4 by $\mathrm{C} 4 \alpha$-demethylase to produce 


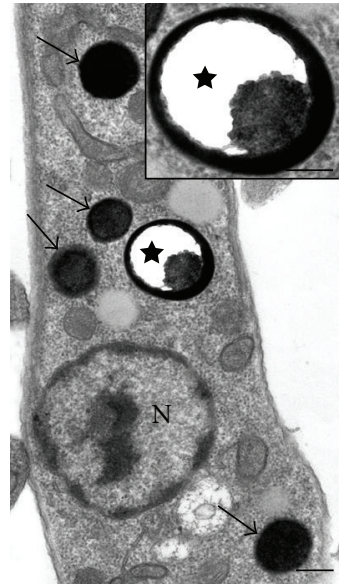

(a)

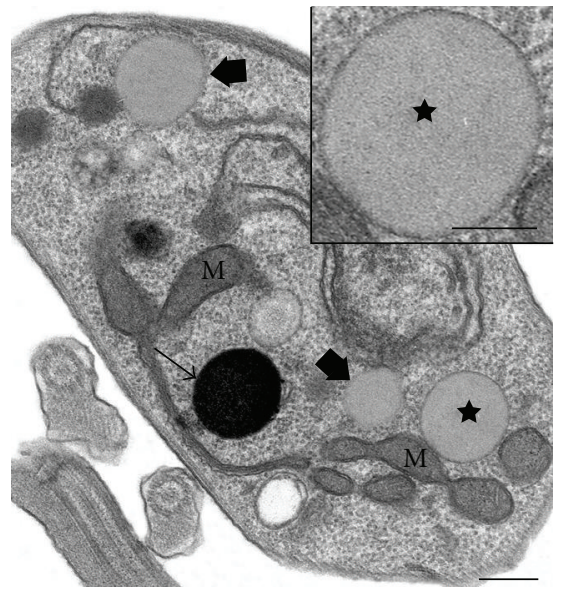

(b)

FIGURE 10: Treatment of promastigotes with ER-119884 induces the accumulation of several lipid droplets in the cytosol, sometimes appearing as an electrondense structure due to osmium tetroxide concentration ((a)-(b), small arrows), and as a classic lipid body surrounded by a phospholipid monolayer ((b), large arrows). At high magnification (stars), it is evident that the structures are completely different, probably indicating a distintic nature of the lipids that accumulate in these inclusions. $\mathrm{M}$, mitochondrion; N, nucleus. All images are reproduced with permission from [37] American Society for Microbiology. Bars, $0.5 \mu \mathrm{m}$.

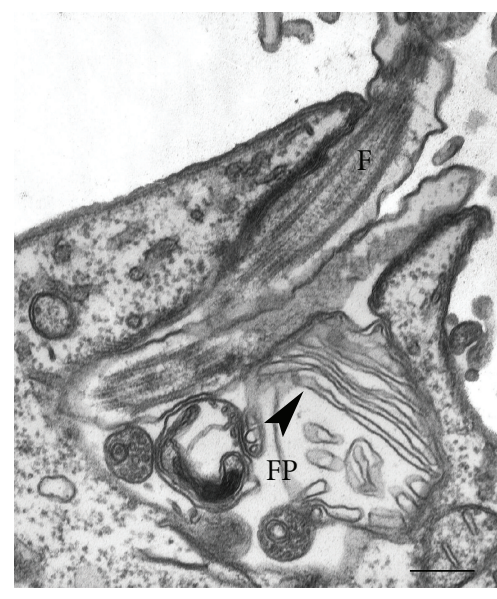

(a)

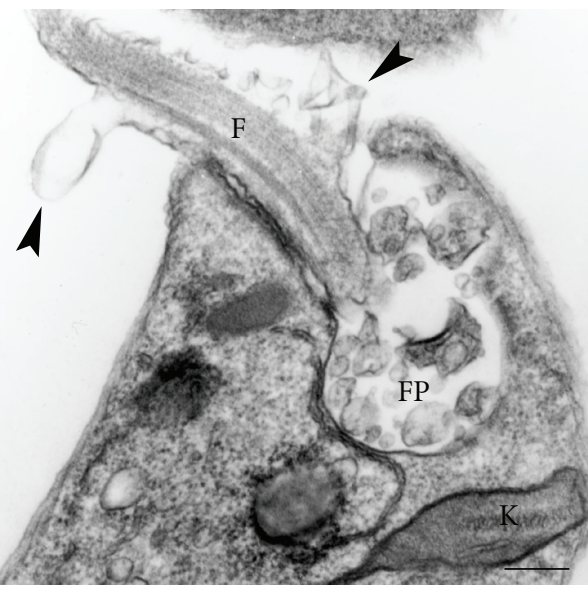

(b)

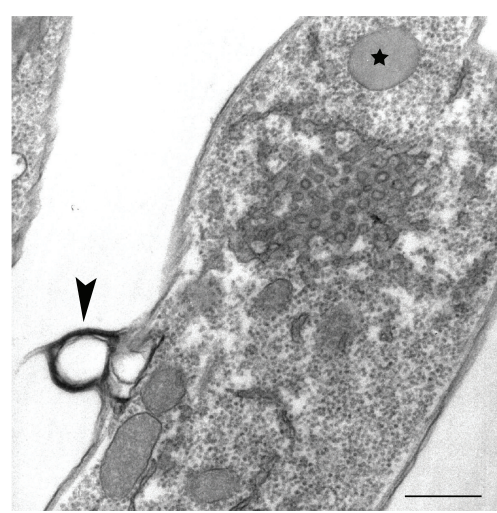

(c)

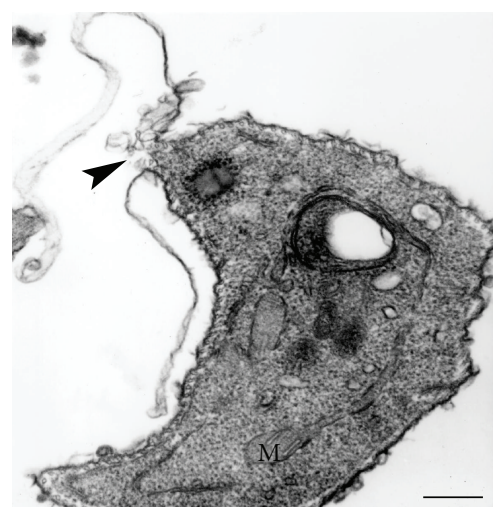

(d)

FIGURE 11: Ultrathin sections of L. amazonensis promastigotes treated with different sterol biosynthesis inhibitors showing severe alterations in the plasma membrane lining (a) the flagellar pocket, (b) the flagellum, and (c)-(d) the cell body. In (d) it is possible to observe a breakdown in the plasma membrane and release of the subpellicular microtubules (arrowhead). Star in (c) shows a classic lipid body. F, flagellum; FP, flagellar pocket; k, kinetoplast; M, mitochondrion. Images are reproduced with permission from [79] (a), and [66] (b), (d) Elsevier. Bars, $0.25 \mu \mathrm{m}(\mathrm{a})-(\mathrm{c})$ and $0.5 \mu \mathrm{m}(\mathrm{d})$. 


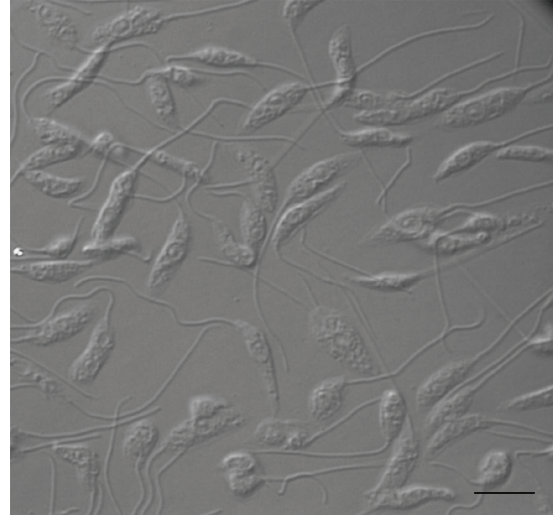

(a)

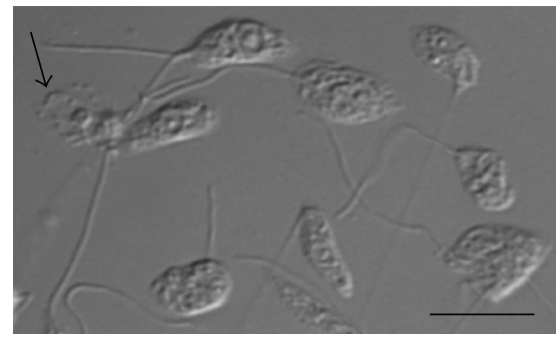

(c)

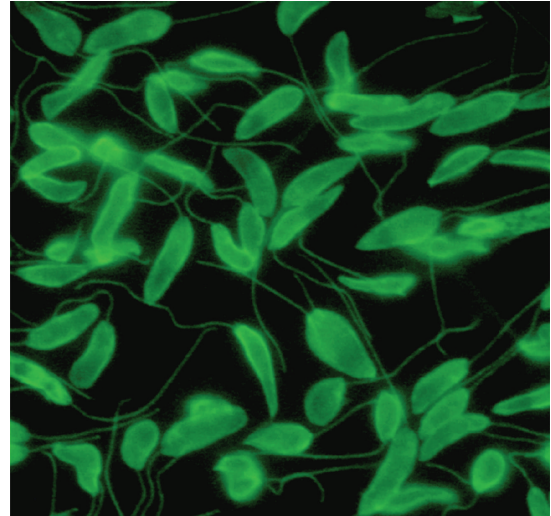

(b)

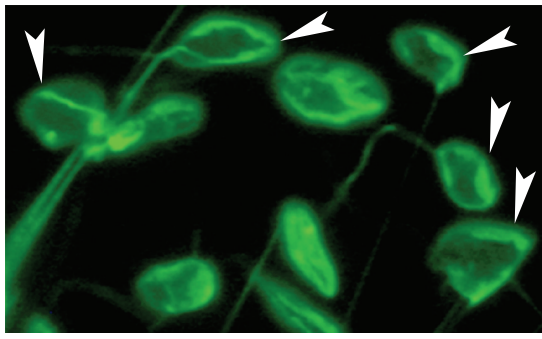

(d)

FIGURE 12: DIC microscopy (left panel) and immunofluorescence microcospy (right panel) of L. amazonensis promastigotes control (a)-(b) and treated with ER-119884 (c)-(d). The labeling corresponds to the cytoskeleton constituted mainly by tubulin, revelead here by using of an Alexa 488-labeled secondary antibody. The black arrow in DIC image corresponds to the cell body which sometimes appeared changed and rounded as compared to the control parasites, while the white arrows point to several tubulin clusters that accumulated in the cytosol after treatment. All images are reproduced with permission from [37] American Society for Microbiology. Bars, $5 \mu \mathrm{m}$.

4-desmethylsterol [11, 46, 100]. In T. cruzi epimastigotes and amastigotes, the accumulating $14 \alpha$-methylsterol is lanosterol, particularly its C-24 alkylation product (24methylenedihydrolanosterol) $[5,14,69,71-75,99,101]$. Unlike Leishmania, demethylation at C4 seems to be very restricted in T. cruzi, and the $\mathrm{C} 4 \alpha$-demethylase has higher specificity for sterols after the removal of the $14 \alpha$-methyl. On the other hand, treatment with SCH 56592 (posaconazole) also causes an accumulation of squalene, possibly indicating that some regulatory step involved in squalene cyclization is regulated by high amounts of lanosterol and its 24-methylene derivatives [72].

The C-24 transmethylation reaction catalyzed by 24SMT in trypanosomatids is inhibited by various azasterols that have a nitrogen substitution in the side chain. This leads to a depletion of $\mathrm{C}_{28}$-sterols such as ergosterol, episterol, and 5-dehydroepisterol, which are then replaced by large amounts of zimosterol and cholesterol ingested from the culture media $[11,42,43,102]$ through the endocytic pathway [3]. Furthermore, a simultaneous incubation with azasterol and ketoconazole induces an accumulation of lanosterol, 4,14-dimethyl-zymosterol, and 14-methyl-zymosterol [11, $42,43]$, indicating that the mechanism of action of 24SMT in trypanosomatids is similar to that observed in fungi, yeasts, and plants $[32,38,44,103-105]$. These results also indicate that in some trypanosomatids, 24-SMT can use zymosterol or its 14-methyl or 4,14-dimethyl derivatives as substrates. In the case of fungi, the substrate for 24-SMT is restricted to zymosterol $[32,38]$. On the other hand, experiments exposing Leishmania spp. to low concentration azasterol for a long time showed that they are able to survive by modulating biosynthesis to use $\mathrm{C}_{27}$-sterols as substrates for 24-SMT [102]. Thus, these biochemical analyses indicate that in the case of sterol biosynthesis it is better to use combination therapy to inhibit more than one step in order to completely eliminate all the sterol substrates, particularly of the 24-SMT enzyme, which participates in essential reactions.

It is important to point out that in all biochemical analyses of the lipid composition of trypanosomatids after drug treatment, a significant accumulation of cholesterol is observed in both T. cruzi and Leishmania spp., indicating that the parasites try to compensate for the absence of endogenous sterols. It is also clear that 24-methyl and/or 24ethyl sterols are essential for the maintenance of membrane structure and function, as well as other vital cellular process like the cell cycle. 

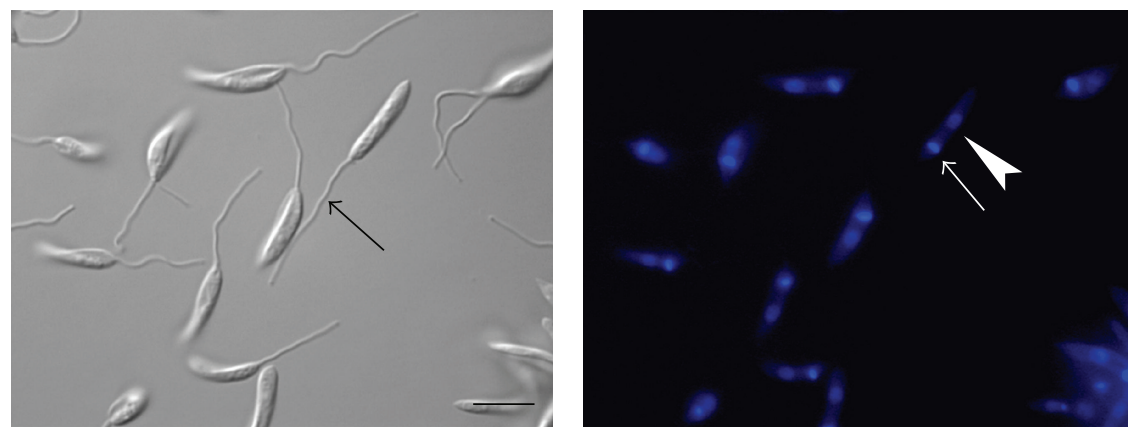

(a)
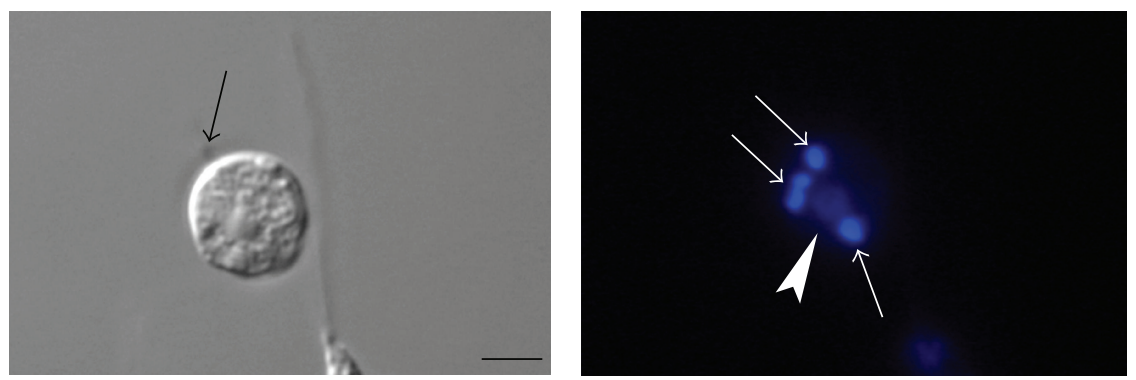

(b)

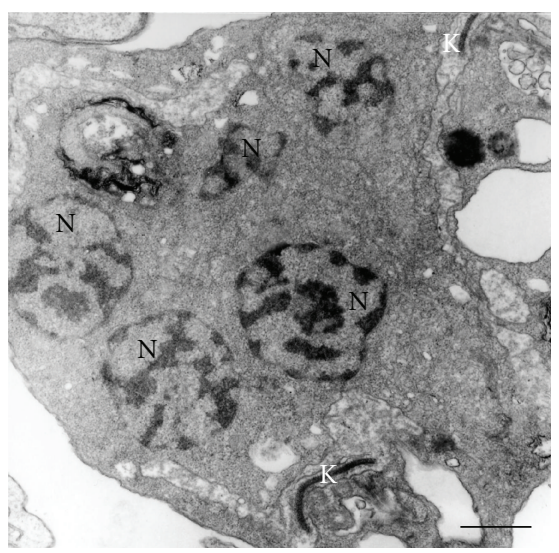

(c)

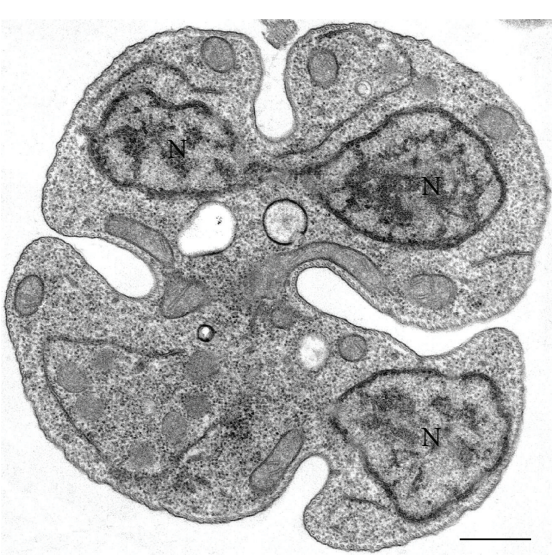

(d)

FIGURE 13: DIC microscopy ((a)-(b), left panel), fluorescence with DAPI ((a)-(b), right panel) and transmission electron microscopy (c)(d) of treated-promastigotes to evidenciate the alterations in the cell cycle after treatment with BPQ-OH and ER-119884. (a) Control cells present a correct number of kinetoplast (white arrow), nucleus (arrowhead), and flagellum (black arrow), one of each for a unique cell. (b) After treatment, the number of these organelles is completely alterated and it is possible to find cells with one flagellum (black arrow), four kinetoplast (white arrows), and one nucleus (arrowhead). (c)-(d) Alterations in the cell cycle can also be evidenced by transmission electron microscopy with the appearance of cells with several nuclei and kinetoplasts. K, kinetoplast; $\mathrm{N}$, nucleus. These images are reproduced with permission from [37] American Society for Microbiology (a), (b), (d), and from [66] Elsevier (c). Bars, $5 \mu \mathrm{m}$ (a)-(b), and 0.5 $\mu \mathrm{m}$ (c)-(d).

\section{Effects of SB Inhibitors in Experimentally Infected Animals and Perspectives for Human Therapy}

Several of the SB inhibitors have been tested using murine models of Chagas' disease, leishmaniasis, and malaria. Treatment of acute Chagas' disease with mevinolin (lovastatin) in combination with ketoconazole led to the elimination of circulating parasites and complete protection against death in the murine model [56].
Bisphosphonates were also tested in acute Chagas' disease as well as in leishmaniasis experimental models. In Leishmania infections, recent studies showed that pamidronate is able to promote a radical cure of experimental cutaneous leishmaniasis in mice [105], and also that it is active in vivo against $L$. donovani by intravenous administration [106]. Furthermore, in vivo studies using the murine model of acute Chagas' disease showed that treatment with $1 \mathrm{mg} / \mathrm{kg}$ risedronate per day for 7 days induced a more than $90 \%$ reduction in parasitemia and significantly increased the animals' survival. On the other hand, at higher concentrations 


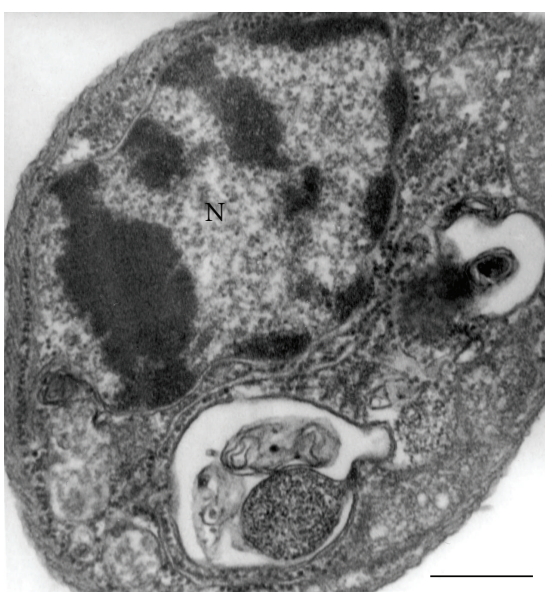

FIgURE 14: Leishmania amazonensis promastigote treated with sterol biosynthesis inhibitors showing a condensation of the nuclear chromatin, a characteristic feature of the apoptosis-like cell death process. $\mathrm{N}$, nucleus. Bar, $0.5 \mu \mathrm{m}$.

(up to $10 \mathrm{mg} / \mathrm{kg}$ per day), risedronate led to a reduction of parasitemia and mortality with no toxic effects for the treated animals [107].

Inhibitors of squalene synthase such as ER-119884 and E5700 were also tested in a murine model of Chagas' disease, revealing that $\mathrm{E} 5700$ is able to provide full protection against death and to completely suppress parasitemia, with no toxicity to the host [36].

Some azoles were also tested against $T$. cruzi and Leishmania spp. with interesting results. D0870, a bis-triazole derivative, showed a potent effect, preventing death and inducing parasitological cure in 70 to $90 \%$ of animals in acute and chronic murine models of Chagas' disease [71, 108]. SCH 56592 (posaconazole), which is one of the new triazole derivatives, has been tested against Chagas' disease [72, 109] and leishmaniasis [110]. In the acute Chagas' disease model, 43 doses of $\geq 10 \mathrm{mg} / \mathrm{kg}$ of body weight/day promoted 85 to $100 \%$ survival, with 90 to $100 \%$ cure of the animals as verified by parasitological, serological and PCR-diagnostics. By contrast, ketoconazole at $30 \mathrm{mg} / \mathrm{kg} /$ day gave only a $60 \%$ survival rate and a $20 \%$ cure rate. [72]. In the chronic phase, the results were also positive, with $85 \%$ of the animals protected from death and 75\% parasitologically cured. In addition, the combination of posaconazole with amiodarone produced a delay in the development of parasitemia after a high rate of infection mice, and in mice with a lower infection rate, the survival and cure were higher when compared with posaconazole alone [73]. Amiodarone alone also led to a decrease in parasitemia and a $40 \%$ increase in the survival of the infected animals [73]. On the other hand, in cutaneous leishmaniasis, treatment with 60 and $30 \mathrm{mg} / \mathrm{kg} /$ day, of SCH 56592 was highly efficacious, and the higher dose was superior to amphotericin B at a dose of $1 \mathrm{mg} / \mathrm{kg} /$ day [110]. In visceral leishmaniasis due to $L$. donovani infection, treated mice showed a significant reduction in parasite burden in the liver and spleen compared to untreated mice [110].
In vivo studies with 22,26-azasterol, one of the most potent inhibitors of 24-SMT, have shown that it has selective antiparasitic activity in a murine model of acute Chagas' disease [43].

\section{Perspectives}

It is clear that the sterol biosynthesis pathway plays a key role in the metabolism of eukaryotic cells. The observation that compounds found in several steps of this metabolic pathway plays essential roles in several basic physiological processes spurred several groups to further analyze this metabolic pathway. From the parasitological point of view, it will be important to test new drugs developed for other medical purposes, such as control of blood cholesterol levels in humans, against trypanosomatids. The same assumption is valid for drugs interfering with the SB pathway in fungal cells. On the other hand, since trypanosomatids, fungi and mammals share several steps of the SB pathway, the trypanosomatids may constitute a useful biological system for the screening of candidate SB inhibitors, and thereby facilitate the search for cholesterol-control drugs for humans. Finally, we expect that new studies will appear providing a better characterization of the last steps of the SB pathway, especially those involved in transformation of zimosterol into ergosterol.

\section{Acknowledgments}

The authors were supported by the Conselho Nacional de Desenvolvimento Científico e Tecnológico (CNPq), Programa de Núcleos de Excelência (PRONEX), Fundação Carlos Chagas Filho de Amparo à Pesquisa do Estado do Rio de Janeiro (FAPERJ), and Coordenação de Aperfeiçoamento de Pessoal de Nível Superior (CAPES). J. C. F. R. has a posdoctoral fellowship from the Coordenação de Aperfeiçoamento de Pessoal de Nível Superior (CAPES). This review is in celebration of the 100th anniversary of Chagas' disease discovery.

\section{References}

[1] L. J. M. Fagundes, Estudo de esteróis e lipídeos totais em Leptomonas pessoai cultivada a $28^{\circ} \mathrm{C}$ e $37^{\circ} \mathrm{C}$, M.S. thesis, Instituto de Microbiologia da Universidade Federal do Rio de Janeiro, Rio de Janeiro, Brazil, 1974.

[2] W. de Souza, C. Sant'anna, and N. L. Cunha-e-Silva, "Electron microscopy and cytochemistry analysis of the endocytic pathway of pathogenic protozoa," Progress in Histochemistry and Cytochemistry, vol. 44, no. 2, pp. 67-124, 2009.

[3] M. J. Soares and W. de Souza, "Endocytosis of goldlabeled proteins and LDL by Trypanosoma cruzi," Parasitology Research, vol. 77, no. 6, pp. 461-468, 1991.

[4] J. A. Urbina, "Lipid biosynthesis pathways as chemotherapeutic targets in kinetoplastid parasites," Parasitology, vol. 114, supplement 1, pp. S91-S99, 1997.

[5] C. W. Roberts, R. McLeod, D. W. Rice, M. Ginger, M. L. Chance, and L. J. Goad, "Fatty acid and sterol metabolism: 
potential antimicrobial targets in apicomplexan and trypanosomatid parasitic protozoa," Molecular and Biochemical Parasitology, vol. 126, no. 2, pp. 129-142, 2003.

[6] S. Halevy and L. Avivi, "Sterols of trypanosomidae," Annals of Tropical Medicine and Parasitology, vol. 60, pp. 439-444, 1966.

[7] H. Dixon, C. D. Ginger, and J. Williamson, "Trypanosome sterols and their metabolic origins," Comparative Biochemistry and Physiology, vol. 41, no. 1, pp. 1-18, 1972.

[8] E. D. Korn, T. von Brand, and E. J. Tobie, "The sterols of Trypanosoma cruzi and Crithidia fasciculata," Comparative Biochemistry and Physiology, vol. 30, no. 4, pp. 601-610, 1969.

[9] P. A. Haughan and L. J. Goad, "Lipid biochemistry of trypanosomatids," in Biochemical Protozoology, G. Coombs and M. North, Eds., pp. 312-328, Taylor \& Francis, London, UK, 1991.

[10] L. J. Goad, G. G. Holz Jr., and D. H. Beach, "Sterols of Leishmania species. Implications for biosynthesis," Molecular and Biochemical Parasitology, vol. 10, no. 2, pp. 161-170, 1984.

[11] J. C. F. Rodrigues, M. Attias, C. Rodriguez, J. A. Urbina, and W. de Souza, "Ultrastructural and biochemical alterations induced by 22,26 -azasterol, a $\Delta^{24(25)}$-sterol methyltransferase inhibitor, on promastigote and amastigote forms of Leishmania amazonensis," Antimicrobial Agents and Chemotherapy, vol. 46, no. 2, pp. 487-499, 2002.

[12] L. J. Goad, J. S. Keithley, J. D. Berman, D. H. Beach, and G. G. Holz, "The sterols of Leishmania promastigotes and amastigotes: possible implications for chemotherapy," in Leishmaniasis, D. T. Hart, Ed., pp. 495-501, Plenum, New York, NY, USA, 1989.

[13] D. H. Beach, L. J. Goad, and G. G. Holz Jr., "Effects of ketoconazole on sterol biosynthesis by Trypanosoma cruzi epimastigotes," Biochemical and Biophysical Research Communications, vol. 136, no. 3, pp. 851-856, 1986.

[14] A. Liendo, G. Visbal, M. M. Piras, R. Piras, and J. A. Urbina, "Sterol composition and biosynthesis in Trypanosoma cruzi amastigotes," Molecular and Biochemical Parasitology, vol. 104, no. 1, pp. 81-91, 1999.

[15] I. Coppens, P. Baudhuin, F. R. Opperdoes, and P. J. Courtoy, "Receptors for the host low density lipoproteins on the hemoflagellate Trypanosoma brucei: purification and involvement in the growth of the parasite," Proceedings of the National Academy of Sciences of the United States of America, vol. 85, no. 18 , pp. 6753-6757, 1988.

[16] L. Gros, V. M. Castillo-Acosta, C. J. Jiménez, et al., "New azasterols against Trypanosoma brucei: role of 24-sterol methyltransferase in inhibitor action," Antimicrobial Agents and Chemotherapy, vol. 50, no. 8, pp. 2595-2601, 2006.

[17] K. R. Matthews, "The developmental cell biology of Trypanosoma brucei," Journal of Cell Science, vol. 118, no. 2, pp. 283-290, 2005.

[18] K. Fenn and K. R. Matthews, "The cell biology of Trypanosoma brucei differentiation," Current Opinion in Microbiology, vol. 10, no. 6, pp. 539-546, 2007.

[19] K. M. Tyler and D. M. Engman, "The life cycle of Trypanosoma cruzi revisited," International Journal for Parasitology, vol. 31, no. 5-6, pp. 472-481, 2001.

[20] M. A. Vannier-Santos, A. Martiny, and W. de Souza, "Cell biology of Leishmania spp.: invading and evading," Current Pharmaceutical Design, vol. 8, no. 4, pp. 297-318, 2002.

[21] P. J. Trocha and D. B. Sprinson, "Location and regulation of early enzymes of sterol biosynthesis in yeast," Archives of Biochemistry and Biophysics, vol. 174, no. 1, pp. 45-51, 1976.
[22] J. Peña-Diaz, A. Montalvetti, C.-L. Flores, et al., "Mitochondrial localization of the mevalonate pathway enzyme 3hydroxy-3-methyl-glutaryl-CoA reductase in the Trypanosomatidae," Molecular Biology of the Cell, vol. 15, no. 3, pp. 1356-1363, 2004.

[23] J. Carrero-Lérida, G. Pérez-Moreno, V. M. Castillo-Acosta, L. M. Ruiz-Pérez, and D. González-Pacanowskda, "Intracellular location of the early steps of the isoprenoid biosynthetic pathway in the trypanosomatids Leishmania major and Trypanosoma brucei," International Journal for Parasitology, vol. 39, no. 3, pp. 307-314, 2009.

[24] G. A. Keller, M. Pazirandeh, and S. Krisans, "3-hydroxy-3methylglutaryl coenzyme A reductase localization in rat liver peroxisomes and microsomes of control and cholestyraminetreated animals: quantitative biochemical and immunoelectron microscopical analyses," Journal of Cell Biology, vol. 103, no. 3, pp. 875-886, 1986.

[25] L. M. Olivier and S. K. Krisans, "Peroxisomal protein targeting and identification of peroxisomal targeting signals in cholesterol biosynthetic enzymes," Biochimica et Biophysica Acta, vol. 1529, no. 1-3, pp. 89-102, 2000.

[26] P. Hugueney, F. Bouvier, A. Badillo, J. Quennemet, A. d'Harlingue, and B. Camara, "Developmental and stress regulation of gene expression for plastid and cytosolic isoprenoid pathways in pepper fruits," Plant Physiology, vol. 111, no. 2, pp. 619-626, 1996.

[27] K. Okada, T. Saito, T. Nakagawa, M. Kawamukai, and Y. Kamiya, "Five geranylgeranyl diphosphate synthases expressed in different organs are localized into three subcellular compartments in Arabidopsis," Plant Physiology, vol. 122, no. 4, pp. 1045-1056, 2000.

[28] N. Cunillera, A. Boronat, and A. Ferrer, "The Arabidopsis thaliana FPS1 gene generates a novel mRNA that encodes a mitochondrial farnesyl-diphosphate synthase isoform," The Journal of Biological Chemistry, vol. 272, no. 24, pp. 1538115388, 1997.

[29] L. M. Olivier, W. Kovacs, K. Masuda, G.-A. Keller, and S. K. Krisans, "Identification of peroxisomal targeting signals in cholesterol biosynthetic enzymes: AA-CoA thiolase, HMGCoA synthase, MPPD, and FPP synthase," Journal of Lipid Research, vol. 41, no. 12, pp. 1921-1935, 2000.

[30] A. Ortiz-Gómez, C. Jiménez, A. M. Estévez, J. CarreroLérida, L. M. Ruiz-Pérez, and D. González-Pacanowska, "Farnesyl diphosphate synthase is a cytosolic enzyme in Leishmania major promastigotes and its overexpression confers resistance to risedronate," Eukaryotic Cell, vol. 5, no. 7, pp. 1057-1064, 2006.

[31] Y. Ling, Z.-H. Li, K. Miranda, E. Oldfield, and S. N. J. Moreno, "The farnesyl-diphosphate/geranylgeranyldiphosphate synthase of Toxoplasma gondii is a bifunctional enzyme and a molecular target of bisphosphonates," The Journal of Biological Chemistry, vol. 282, no. 42, pp. 3080430816, 2007.

[32] A. C. Oehlschlager and E. Czyzewska, "Rationally designed inhibitors of sterol biosynthesis," in Emerging Targets in Antibacterial and Antifungal Chemotherapy, J. Sutcliffe and N. H. Georgopapadakou, Eds., pp. 437-475, Chapman \& Hall, New York, NY, USA, 1992.

[33] J. F. Thompson, D. E. Danley, S. Mazzalupo, P. M. Milos, M. E. Lira, and H. J. Harwood Jr., "Truncation of human squalene synthase yields active, crystallizable protein," Archives of Biochemistry and Biophysics, vol. 350, no. 2, pp. 283-290, 1998. 
[34] F. R. Opperdoes and J.-P. Szikora, "In silico prediction of the glycosomal enzymes of Leishmania major and trypanosomes," Molecular and Biochemical Parasitology, vol. 147, no. 2, pp. 193-206, 2006.

[35] J. A. Urbina, J. L. Concepcion, S. Rangel, G. Visbal, and R. Lira, "Squalene synthase as a chemotherapeutic target in Trypanosoma cruzi and Leishmania mexicana," Molecular and Biochemical Parasitology, vol. 125, no. 1-2, pp. 35-45, 2002.

[36] J. A. Urbina, J. L. Concepcion, A. Caldera, et al., "In vitro and in vivo activities of E5700 and ER-119884, two novel orally active squalene synthase inhibitors, against Trypanosoma cruzi," Antimicrobial Agents and Chemotherapy, vol. 48, no. 7, pp. 2379-2387, 2004.

[37] J. C. F. Rodrigues, J. L. Concepcion, C. Rodrigues, A. Caldera, J. A. Urbina, and W. de Souza, "In vitro activities of ER119884 and E5700, two potent squalene synthase inhibitors, against Leishmania amazonensis: antiproliferative, biochemical, and ultrastructural effects," Antimicrobial Agents and Chemotherapy, vol. 52, no. 11, pp. 4098-4114, 2008.

[38] K. Barrett-Bee and N. Ryder, "Biochemical aspects of ergosterol biosynthesis inhibition," in Emerging Targets in Antibacterial and Antifungal Chemotherapy, J. Sutcliffe and N. H. Georgopapadakou, Eds., pp. 410-436, Chapman \& Hall, New York, NY, USA, 1992.

[39] K. Ishida, J. C. F. Rodrigues, M. D. Ribeiro, et al., "Growth inhibition and ultrastructural alterations induced by $\Delta^{24(25)}$ sterol methyltransferase inhibitors in Candida spp. isolates, including non-albicans organisms," BMC Microbiology, vol. 9, article 74, 2009.

[40] Y. Aoyama, Y. Yoshida, and R. Sato, "Yeast cytochrome P450 catalyzing lanosterol $14 \alpha$-demethylation. II. Lanosterol metabolism by purified P-450(14DM) and by intact microsomes," The Journal of Biological Chemistry, vol. 259, no. 3, pp. 1661-1666, 1984.

[41] N. S. Ryder, G. Seidl, and P. F. Troke, "Effect of the antimycotic drug naftifine on growth of and sterol biosynthesis in Candida albicans," Antimicrobial Agents and Chemotherapy, vol. 25, no. 4, pp. 483-487, 1984.

[42] J. A. Urbina, J. Vivas, G. Visbal, and L. M. Contreras, "Modification of the sterol composition of Trypanosoma (Schizotrypanum) cruzi epimastigotes by $\Delta^{24(25)}$-sterol methyl transferase inhibitors and their combinations with ketoconazole," Molecular and Biochemical Parasitology, vol. 73, no. 1-2, pp. 199-210, 1995.

[43] J. A. Urbina, J. Vivas, K. Lazardi, et al., "Antiproliferative effects of $\Delta^{24(25)}$ sterol methyl transferase inhibitors on Trypanosoma (Schizotrypanum) cruzi: in vitro and in vivo studies," Chemotherapy, vol. 42, no. 4, pp. 294-307, 1996.

[44] A. Liendo, G. Visbal, M. M. Piras, R. Piras, and J. A. Urbina, "Sterol composition and biosynthesis in Trypanosoma cruzi amastigotes," Molecular and Biochemical Parasitology, vol. 104, no. 1, pp. 81-91, 1999.

[45] K. Barrett-Bee, L. Newboult, and P. Pinder, "Biochemical changes associated with the antifungal action of the triazole ICI 153,066 on Candida albicans and Trichophyton quinckeanum," FEMS Microbiology Letters, vol. 79, no. 2-3, pp. 127131, 1991.

[46] D. H. Beach, L. J. Goad, and G. G. Holz Jr., "Effects of antimycotic azoles on growth and sterol biosynthesis of Leishmania promastigotes," Molecular and Biochemical Parasitology, vol. 31, no. 2, pp. 149-162, 1988.

[47] C. Jiménez-Jiménez, J. Carrero-Lérida, M. Sealey-Cardona, L. M. Ruiz-Pérez, J. A. Urbina, and D. González-Pacanowska, " $\Delta^{24(25)}$-sterol methenyltransferase: intracellular localization and azasterol sensitivity in Leishmania major promastigotes overexpressing the enzyme," Molecular and Biochemical Parasitology, vol. 160, no. 1, pp. 52-59, 2008.

[48] S. A. Doggrell, "Is atorvastatin superior to other statins? Analysis of the clinical trials with atorvastatin having cardiovascular endpoints," Reviews on Recent Clinical Trials, vol. 1, no. 2, pp. 143-153, 2006.

[49] G. A. Rodan, "Mechanisms of action of bisphosphonates," Annual Review of Pharmacology and Toxicology, vol. 38, pp. 375-388, 1998.

[50] D. González-Pacanowska, B. Arison, C. M. Havel, and J. A. Watson, "Isopentenoid synthesis in isolated embryonic Drosophila cells. Farnesol catabolism and w-oxidation," The Journal of Biological Chemistry, vol. 263, no. 3, pp. 13011306, 1988.

[51] G. R. Brown, D. S. Clarke, A. J. Foubister, et al., "Synthesis and activity of a novel series of 3-biarylquinuclidine squalene synthase inhibitors," Journal of Medicinal Chemistry, vol. 39, no. 15, pp. 2971-2979, 1996.

[52] F. McTaggart, G. R. Brown, R. G. Davidson, et al., "Inhibition of squalene synthase of rat liver by novel 3' substituted quinuclidines," Biochemical Pharmacology, vol. 51, no. 11, pp. 1477-1487, 1996.

[53] V. Charlton-Menys and P. N. Durrington, "Squalene synthase inhibitors: clinical pharmacology and cholesterol-lowering potential," Drugs, vol. 67, no. 1, pp. 11-16, 2007.

[54] J. A. Urbina, G. Visbal, L. M. Contreras, G. McLaughlin, and R. Docampo, "Inhibitors of $\Delta^{24(25)}$ sterol methyltransferase block sterol synthesis and cell proliferation in Pneumocystis carinii," Antimicrobial Agents and Chemotherapy, vol. 41, no. 7, pp. 1428-1432, 1997.

[55] G. Visbal, A. Alvarez, B. Moreno, and G. San-Blas, " $S$ adenosyl-L-methionine inhibitors $\Delta^{24}$-sterol methyltransferase and $\Delta^{24(28)}$-sterol methylreductase as possible agents against Paracoccidioides brasiliensis," Antimicrobial Agents and Chemotherapy, vol. 47, no. 9, pp. 2966-2970, 2003.

[56] J. A. Urbina, K. Lazardi, E. Marchan, et al., "Mevinolin (lovastatin) potentiates the antiproliferative effects of ketoconazole and terbinafine against Trypanosoma (Schizotrypanum) cruzi: in vitro and in vivo studies," Antimicrobial Agents and Chemotherapy, vol. 37, no. 3, pp. 580-591, 1993.

[57] R. Docampo and S. N. Moreno, "Bisphosphonates as chemotherapeutic agents against trypanosomatid and apicomplexan parasites," Current Drug Targets-Infectious Disorders, vol. 1, no. 1, pp. 51-61, 2001.

[58] R. Docampo and S. N. J. Moreno, "The acidocalcisome as a target for chemotherapeutic agents in protozoan parasites," Current Pharmaceutical Design, vol. 14, no. 9, pp. 882-888, 2008.

[59] M. B. Martin, J. S. Grimley, J. C. Lewis, et al., "Bisphosphonates inhibit the growth of Trypanosoma brucei, Trypanosoma cruzi, Leishmania donovani, Toxoplasma gondii, and Plasmodium falciparum: a potential route to chemotherapy," Journal of Medicinal Chemistry, vol. 44, no. 6, pp. 909-916, 2001.

[60] J. M. Sanders, Y. Song, J. M. W. Chan, et al., "Pyridinium-1yl bisphosphonates are potent inhibitors of farnesyl diphosphate synthase and bone resorption," Journal of Medicinal Chemistry, vol. 48, no. 8, pp. 2957-2963, 2005.

[61] S. H. Szajnman, G. E. García Liñares, Z.-H. Li, et al., "Synthesis and biological evaluation of 2-alkylaminoethyl1,1-bisphosphonic acids against Trypanosoma cruzi and Toxoplasma gondii targeting farnesyl diphosphate synthase," Bioorganic and Medicinal Chemistry, vol. 16, no. 6, pp. 32833290, 2008. 
[62] L. R. Garzoni, A. Caldera, M. D. N. L. Meirelles, et al., "Selective in vitro effects of the farnesyl pyrophosphate synthase inhibitor risedronate on Trypanosoma cruzi," International Journal of Antimicrobial Agents, vol. 23, no. 3, pp. 273-285, 2004.

[63] J. D. Bergstrom, C. Dufresne, G. F. Bills, M. Nallin-Omstead, and K. Byrne, "Discovery, biosynthesis, and mechanism of action of the zaragozic acids: potent inhibitors of squalene synthase," Annual Review of Microbiology, vol. 49, pp. 607639, 1995.

[64] M. V. Braga, J. A. Urbina, and W. de Souza, "Effects of squalene synthase inhibitors on the growth and ultrastructure of Trypanosoma cruzi," International Journal of Antimicrobial Agents, vol. 24, no. 1, pp. 72-78, 2004.

[65] S. O. Lorente, R. Gómez, C. Jiménez-Jiménez, et al., "Biphenylquinuclidines as inhibitors of squalene synthase and growth of parasitic protozoa," Bioorganic and Medicinal Chemistry, vol. 13, no. 10, pp. 3519-3529, 2005.

[66] J. C. F. Rodrigues, J. A. Urbina, and W. de Souza, "Antiproliferative and ultrastructural effects of BPQ-OH, a specific inhibitor of squalene synthase, on Leishmania amazonensis," Experimental Parasitology, vol. 111, no. 4, pp. 230-238, 2005.

[67] S. B. Cammerer, C. Jiménez-Jiménez, S. Jones, et al., "Quinuclidine derivatives as potential antiparasitics," Antimicrobial Agents and Chemotherapy, vol. 51, no. 11, pp. 4049-4061, 2007.

[68] M. Sealey-Cardona, S. Cammerer, S. Jones, et al., "Kinetic characterization of squalene synthase from Trypanosoma cruzi: selective inhibition by quinuclidine derivatives," Antimicrobial Agents and Chemotherapy, vol. 51, no. 6, pp. 2123-2129, 2007.

[69] D. H. Beach, L. J. Goad, and G. G. Holz Jr., "Effects of ketoconazole on sterol biosynthesis by Trypanosoma cruzi epimastigotes," Biochemical and Biophysical Research Communications, vol. 136, no. 3, pp. 851-856, 1986.

[70] M. A. Vannier-Santos, J. A. Urbina, A. Martiny, A. Neves, and W. de Souza, "Alterations induced by the antifungal compounds ketoconazole and terbinafine in Leishmania," The Journal of Eukaryotic Microbiology, vol. 42, no. 4, pp. 337346, 1995.

[71] J. A. Urbina, G. Payares, J. Molina, et al., "Cure of shortand long-term experimental Chagas' disease using D0870," Science, vol. 273, no. 5277, pp. 969-971, 1996.

[72] J. A. Urbina, G. Payares, L. M. Contreras, et al., "Antiproliferative effects and mechanism of action of SCH 56592 against Trypanosoma (Schizotrypanum) cruzi: in vitro and in vivo studies," Antimicrobial Agents and Chemotherapy, vol. 42, no. 7, pp. 1771-1777, 1998.

[73] G. Benaim, J. M. Sanders, Y. Garcia-Marchán, et al., "Amiodarone has intrinsic anti-Trypanosoma cruzi activity and acts synergistically with posaconazole," Journal of Medicinal Chemistry, vol. 49, no. 3, pp. 892-899, 2006.

[74] K. Lazardi, J. A. Urbina, and W. de Souza, "Ultrastructural alterations induced by two ergosterol biosynthesis inhibitors, ketoconazole and terbinafine, on epimastigotes and amastigotes of Trypanosoma (Schizotrypanum) cruzi," Antimicrobial Agents and Chemotherapy, vol. 34, no. 11, pp. 2097-2105, 1990.

[75] K. Lazardi, J. A. Urbina, and W. de Souza, "Ultrastructural alterations induced by ICI 195,739, a bis-triazole derivative with strong antiproliferative action against Trypanosoma (Schizotrypanum) cruzi," Antimicrobial Agents and Chemotherapy, vol. 35, no. 4, pp. 736-740, 1991.
[76] J. Vivas, J. A. Urbina, and W. de Souza, "Ultrastructural alterations in Trypanosoma (Schizotrypanum) cruzi induced by $\Delta^{24(25)}$ sterol methyl transferase inhibitors and their combinations with ketoconazole," International Journal of Antimicrobial Agents, vol. 7, no. 4, pp. 235-240, 1996.

[77] X. Serrano-Martín, Y. García-Marchan, A. Fernandez, et al., "Amiodarone destabilizes intracellular $\mathrm{Ca}^{2+}$ homeostasis and biosynthesis of sterols in Leishmania mexicana," Antimicrobial Agents and Chemotherapy, vol. 53, no. 4, pp. 1403-1410, 2009.

[78] F. Magaraci, C. J. Jiménez, C. Rodrigues, et al., "Azasterols as inhibitors of sterol 24-methyltransferase in Leishmania species and Trypanosoma cruzi," Journal of Medicinal Chemistry, vol. 46, no. 22, pp. 4714-4727, 2003.

[79] J. C. F. Rodrigues, C. F. Bernardes, G. Visbal, J. A. Urbina, A. E. Vercesi, and W. de Souza, "Sterol methenyl transferase inhibitors alter the ultrastructure and function of the Leishmania amazonensis mitochondrion leading to potent growth inhibition," Protist, vol. 158, no. 4, pp. 447-456, 2007.

[80] S. O. Lorente, J. C. F. Rodrigues, C. J. Jiménez, et al., "Novel azasterols as potential agents for treatment of leishmaniasis and trypanosomiasis," Antimicrobial Agents and Chemotherapy, vol. 48, no. 8, pp. 2937-2950, 2004.

[81] A. C. Granthon, M. V. Braga, J. C. F. Rodrigues, et al., "Alterations on the growth and ultrastructure of Leishmania chagasi induced by squalene synthase inhibitors," Veterinary Parasitology, vol. 146, no. 1-2, pp. 25-34, 2007.

[82] W. de Souza, M. Attias, and J. C. F. Rodrigues, "Particularities of mitochondrial structure in parasitic protozoa (Apicomplexa and Kinetoplastida)," submitted to The International Journal of Biochemistry \& Cell Biology.

[83] I. V. Palmié-Peixoto, M. R. Rocha, J. A. Urbina, W. de Souza, M. Einicker-Lamas, and M. C. M. Motta, "Effects of sterol biosynthesis inhibitors on endosymbiont-bearing try panosomatids," FEMS Microbiology Letters, vol. 255, no. 1, pp. 33-42, 2006.

[84] C. O. Rodrigues, R. Catisti, S. A. Uyemura, et al., "The sterol composition of Trypanosoma cruzi changes after growth in different culture media and results in different sensitivity to digitonin-permeabilization," Journal of Eukaryotic Microbiology, vol. 48, no. 5, pp. 588-594, 2001.

[85] W. de Souza and D. P. Cavalcanti, "DNA containing organelles in pathogenic protozoa," Trends in Cell \& Molecular Biology, vol. 2, pp. 89-104, 2008.

[86] J. F. R. Kerr, "History of the events leading to the formulation of the apoptosis concept," Toxicology, vol. 181-182, pp. 471474, 2002.

[87] J. C. F. Rodrigues and W. de Souza, "Ultrastructural alterations in organelles of parasitic protozoa induced by different classes of metabolic inhibitos," Current Pharmaceutical Design, vol. 14, no. 9, pp. 925-938, 2008.

[88] R. F. S. Menna-Barreto, K. Salomão, A. P. Dantas, et al., "Different cell death pathways induced by drugs in Trypanosoma cruzi: an ultrastructural study," Micron, vol. 40, no. 2, pp. 157-168, 2009.

[89] R. Docampo, W. de Souza, K. Miranda, P. Rohloff, and S. N. J. Moreno, "Acidocalcisomes-conserved from bacteria to man," Nature Reviews Microbiology, vol. 3, no. 3, pp. 251-261, 2005.

[90] J. A. Urbina, S. Pekerar, H.-B. Le, J. Patterson, B. Montez, and E. Oldfield, "Molecular order and dynamics of phosphatidylcholine bilayer membranes in the presence of cholesterol, ergosterol and lanosterol: a comparative study using $2 \mathrm{H}-$, 
13C- and 31P-NMR spectroscopy," Biochimica et Biophysica Acta, vol. 1238, no. 2, pp. 163-176, 1995.

[91] K. Zhang, M. Showalter, J. Revollo, F.-F. Hsu, J. Turk, and S. M. Beverley, "Sphingolipids are essential for differentiation but not growth in Leishmania," The EMBO Journal, vol. 22, no. 22, pp. 6016-6026, 2003.

[92] K. Zhang, F.-F. Hsu, D. A. Scott, R. Docampo, J. Turk, and S. M. Beverley, "Leishmania salvage and remodelling of host sphingolipids in amastigote survival and acidocalcisome biogenesis," Molecular Microbiology, vol. 55, no. 5, pp. 15661578, 2005.

[93] M. L. Salto, L. E. Bertello, M. Vieira, R. Docampo, S. N. J. Moreno, and R. M. de Lederkremer, "Formation and remodeling of inositolphosphoceramide during differentiation of Trypanosoma cruzi from trypomastigote to amastigote," Eukaryotic Cell, vol. 2, no. 4, pp. 756-768, 2003.

[94] A. P. Dantas, H. S. Barbosa, and S. L. de Castro, "Biological and ultrastructural effects of the anti-microtubule agent taxol against Trypanosoma cruzi," Journal of Submicroscopic Cytology and Pathology, vol. 35, no. 3, pp. 287-294, 2003.

[95] K. Gull, "The cytoskeleton of trypanosomatid parasites," Annual Review of Microbiology, vol. 53, pp. 629-655, 1999.

[96] C. Dahl, H.-P. Biemann, and J. Dahl, "A protein kinase antigenically related to $\mathrm{pp} 60^{\mathrm{v} \text {-src }}$ possibly involved in yeast cell cycle control: positive in vivo regulation by sterol," Proceedings of the National Academy of Sciences of the United States of America, vol. 84, no. 12, pp. 4012-4016, 1987.

[97] R. F. Gaber, D. M. Copple, B. K. Kennedy, M. Vidal, and M. Bard, "The yeast gene ERG6 is required for normal membrane function but is not essential for biosynthesis of the cell-cycle-sparking sterol," Molecular and Cellular Biology, vol. 9, no. 8, pp. 3447-3456, 1989.

[98] L. J. Goad, G. G. Holz Jr., and D. H. Beach, "Effect of the allylamine antifungal drug SF 86-327 on the growth and sterol synthesis of Leishmania mexicana mexicana promastigotes," Biochemical Pharmacology, vol. 34, no. 20, pp. 3785-3788, 1985.

[99] J. A. Urbina, K. Lazardi, T. Aguirre, M. M. Piras, and R. Piras, "Antiproliferative synergism of the allylamine SF 86327 and ketoconazole on epimastigotes and amastigotes of Trypanosoma (Schizotrypanum) cruzi," Antimicrobial Agents and Chemotherapy, vol. 32, no. 8, pp. 1237-1242, 1988.

[100] L. J. Goad, G. G. Holz Jr., and D. H. Beach, "Sterols of ketoconazole-inhibited Leishmania mexicana mexicana promastigotes," Molecular and Biochemical Parasitology, vol. 15, no. 3, pp. 257-279, 1985.

[101] L. J. Goad, R. L. Berens, J. J. Marr, D. H. Beach, and G. G. Holz Jr., "The activity of ketoconazole and other azoles against Trypanosoma cruzi: biochemistry and chemotherapeutic action in vitro," Molecular and Biochemical Parasitology, vol. 32, no. 2-3, pp. 179-190, 1989.

[102] P. A. Haughan, M. L. Chance, and L. J. Goad, "Effects of an azasterol inhibitor of sterol 24-transmethylation an sterol biosynthesis and growth of Leishmania donovani promastigotes," Biochemical Journal, vol. 308, no. 1, pp. 3138, 1995.

[103] E. I. Mercer, "Inhibitors of sterol biosynthesis and their applications," Progress in Lipid Research, vol. 32, no. 4, pp. 357-416, 1993.

[104] A. Rahur, M. Taton, and P. Benveniste, "Inhibition of sterol biosynthesis in higher plants by analogues of high energy carbocationic intermediates," in Biochemistry of Cell Walls and Membranes in Fungi, P. J. Kuhn, A. P. J. Trinci, M. J.
Jung, M. W. Goosey, and L. G. Copping, Eds., pp. 206-221, Springer, Berlin, Germany, 1990.

[105] N. Rodriguez, B. N. Bailey, M. B. Martin, E. Oldfield, J. A. Urbina, and R. Docampo, "Radical cure of experimental cutaneous leishmaniasis by the bisphosphonate pamidronate," Journal of Infectious Diseases, vol. 186, no. 1, pp. 138-140, 2002.

[106] V. Yardley, A. A. Khan, M. B. Martin, et al., "In vivo activities of farnesyl pyrophosphate synthase inhibitors against Leishmania donovani and Toxoplasma gondii," Antimicrobial Agents and Chemotherapy, vol. 46, no. 3, pp. 929-931, 2002.

[107] L. R. Garzoni, M. C. Waghabi, M. M. Baptista, et al., "Antiparasitic activity of risedronate in a murine model of acute Chagas' disease," International Journal of Antimicrobial Agents, vol. 23, no. 3, pp. 286-290, 2004.

[108] J. Molina, Z. Brener, A. J. Romanha, and J. A. Urbina, "In vivo activity of the bis-triazole D0870 against drug-susceptible and drug-resistant strains of the protozoan parasite Trypanosoma cruzi," Journal of Antimicrobial Chemotherapy, vol. 46, no. 1, pp. 137-140, 2000.

[109] J. Molina, O. Martins-Filho, Z. Brener, A. J. Romanha, D. Loebenberg, and J. A. Urbina, "Activities of the triazole derivative SCH 56592 (Posaconazole) against drug-resistant strains of the protozoan parasite Trypanosoma (Schizotrypanum) cruzi in immunocompetent and immunosuppressed murine hosts," Antimicrobial Agents and Chemotherapy, vol. 44, no. 1, pp. 150-155, 2000.

[110] H. M. Al-Abdely, J. R. Graybill, D. Loebenberg, and P. C. Melby, "Efficacy of the triazole SCH 56592 against Leishmania amazonensis and Leishmania donovani in experimental murine cutaneous and visceral leishmaniases," Antimicrobial Agents and Chemotherapy, vol. 43, no. 12, pp. 2910-2914, 1999. 


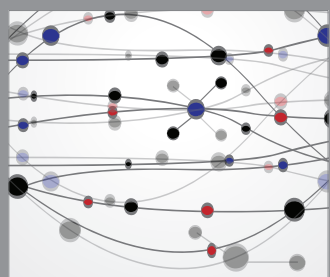

The Scientific World Journal
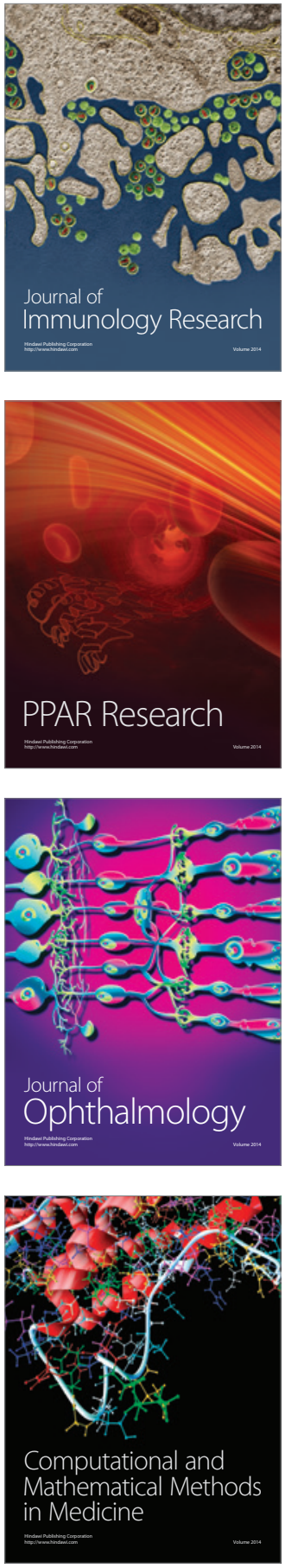

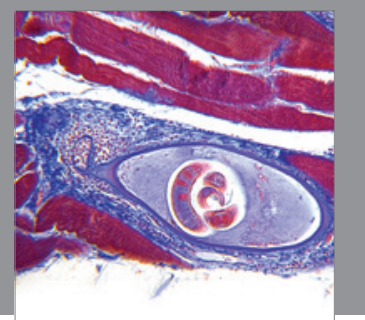

Gastroenterology

Research and Practice
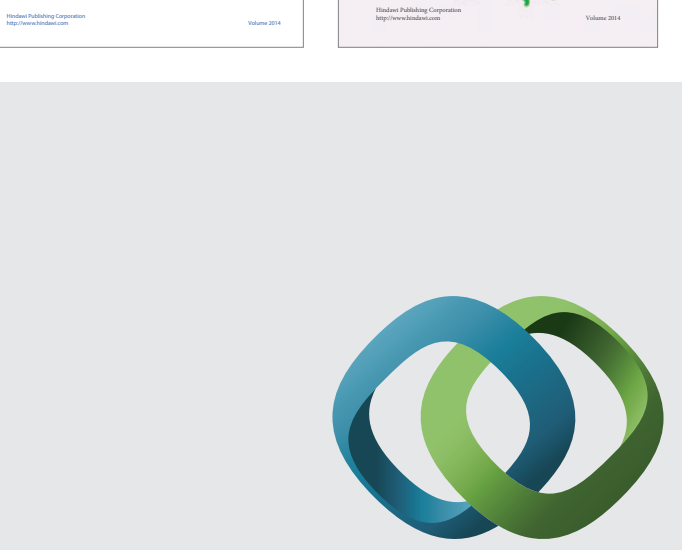

\section{Hindawi}

Submit your manuscripts at

http://www.hindawi.com
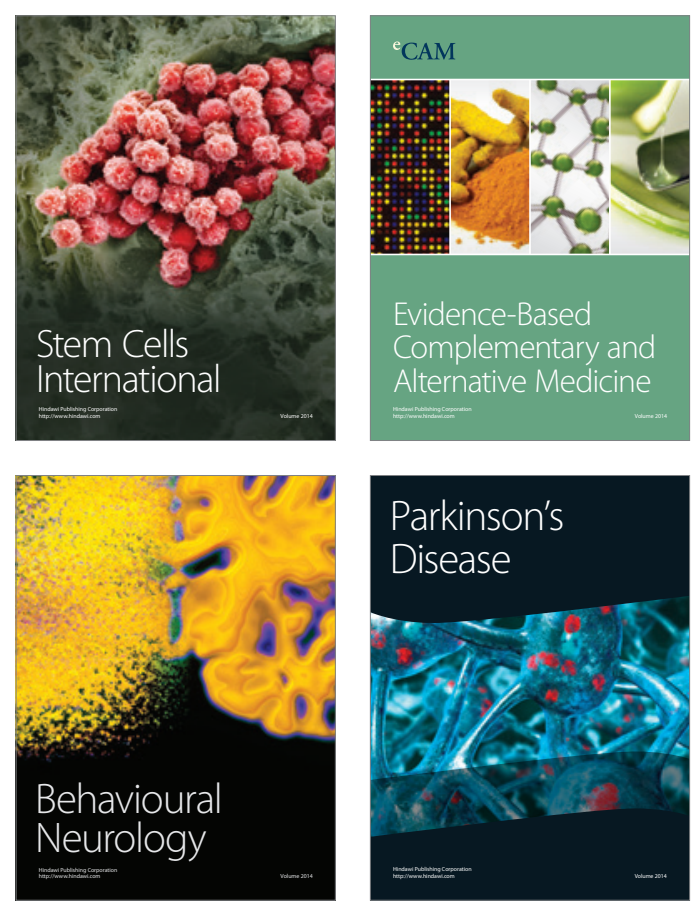

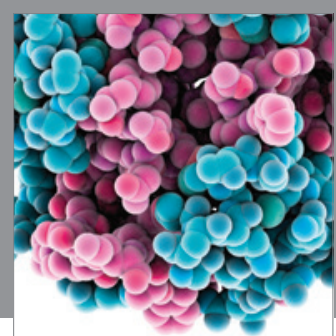

Journal of
Diabetes Research

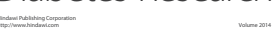

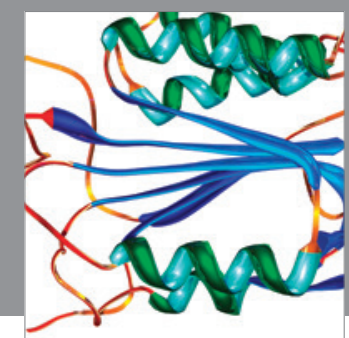

Disease Markers
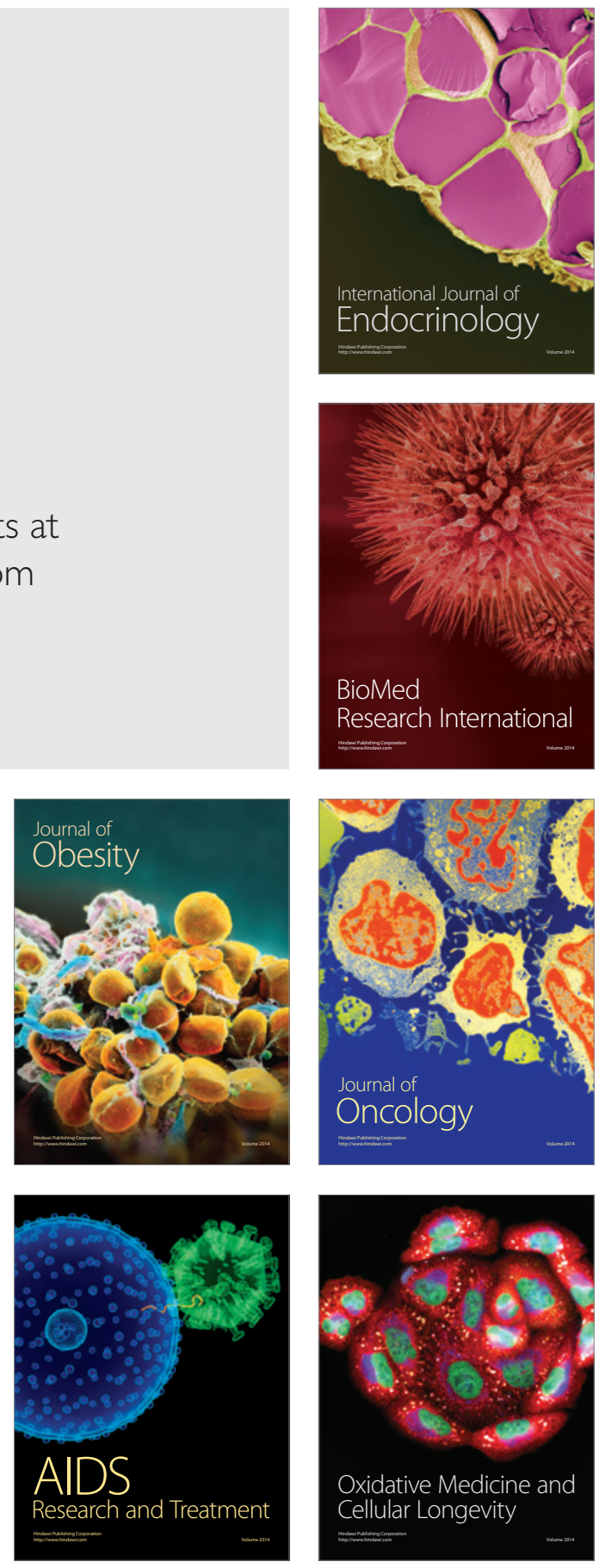